\title{
Dissipative Solutions to the Stochastic Euler Equations
}

\author{
D. Breit(D and T. C. Moyo \\ Communicated by F. Flandoli
}

\begin{abstract}
We study the three-dimensional incompressible Euler equations subject to stochastic forcing. We develop a concept of dissipative martingale solutions, where the nonlinear terms are described by generalised Young measures. We construct these solutions as the vanishing viscosity limit of solutions to the corresponding stochastic Navier-Stokes equations. This requires a refined stochastic compactness method incorporating the generalised Young measures. As a main novelty, our solutions satisfy a form of the energy inequality which gives rise to a weak-strong uniqueness result (pathwise and in law). A dissipative martingale solution coincides (pathwise or in law) with the strong solution as soon as the latter exists.
\end{abstract}

Mathematics Subject Classification. 60H15, 35R60, 76B03, 35Q31.

Keywords. Stochastic Euler equations, Weak-strong uniqueness, Martingale solutions, Vanishing viscosity.

\section{Introduction}

We are interested in the stochastic Euler equations describing the motion of an incompressible inviscid fluid in the three-dimensional torus $\mathbb{T}^{3}$. The flow is described by the velocity field $\mathbf{u}: Q_{T} \rightarrow \mathbb{R}^{3}, Q_{T}=$ $(0, T) \times \mathbb{T}^{3}$, and the pressure $\pi: Q_{T} \rightarrow \mathbb{R}$ and the equations in question read as

$$
\begin{cases}\mathrm{d} \mathbf{u}=-(\nabla \mathbf{u}) \mathbf{u} \mathrm{d} t-\nabla \pi \mathrm{d} t+\Phi \mathrm{d} W & \text { in } Q_{T}, \\ \operatorname{div} \mathbf{u}=0 & \text { in } Q_{T},\end{cases}
$$

subject to periodic boundary conditions for $\mathbf{u}$. The first equation in (1.1) is forced by a cylindrical Wiener process $W$ and $\Phi$ is a Hilbert-Schmidt operator, see Sect. 2.3 for details. Stochastic forces in the equations of motion are frequently used to model phenomena in turbulent flows at high Reynolds number, see e.g. $[20,33,35]$.

As in the deterministic case smooth solutions to (1.1) are only known to exist locally in time, see $[23,28,32]$. The life space of these solutions is an a.s. positive stopping time. While better results are known in the two-dimensional situation, cf. $[2,11,14,27]$, the existence and uniqueness of global strong solutions is a major open problem. In the deterministic case a series of counter examples concerning uniqueness of solutions to the Euler equations have been accomplished recently. These solutions are called wild solutions and are constructed by the method of convex integration pioneered by the work of De Lellis and Székelyhidi [16,17]. As shown in [5] stochastic forces do not seem to change the situation.

In view of these examples one may expect that singularities occur in the long-run and that solutions are not unique. A natural approach to deal with such situations is the concept of measure-valued solutions as introduced by Di Perna and Majda [19] (see also [18]). These solutions are constructed by compactness methods and the nonlinearities are described by generalised Young measures. A generalised Young measure is a triplet $\mathcal{V}=\left(\nu_{t, x}, \nu_{t, x}^{\infty}, \lambda\right)$ consisting of the oscillation measure $\nu_{t, x}$ (a parametrised probability measure on $\mathbb{R}^{3}$ ), the concentration measure $\lambda$ (a non-negative Radon measure on $Q_{T}$ ) and the concentration angle $\nu_{t, x}^{\infty}$ (a parametrised probability measure on the unit sphere $\mathbb{S}^{2}$ ). The convective 
term can be written as the space-time distribution

$$
\mathrm{d}\left\langle\nu_{t, x}, \boldsymbol{\xi} \otimes \boldsymbol{\xi}\right\rangle \mathrm{d} x \mathrm{~d} t+\mathrm{d}\left\langle\nu_{t, x}^{\infty}, \boldsymbol{\xi} \otimes \boldsymbol{\xi}\right\rangle \mathrm{d} \lambda,
$$

where $\boldsymbol{\xi}$ is the dummy variable and

$$
\left\langle\nu_{t, x}, \boldsymbol{\xi} \otimes \boldsymbol{\xi}\right\rangle:=\int_{\mathbb{R}^{3}} \boldsymbol{\xi} \otimes \boldsymbol{\xi} \mathrm{d} \nu_{t, x}(\boldsymbol{\xi}), \quad\left\langle\nu_{t, x}^{\infty}, \boldsymbol{\xi} \otimes \boldsymbol{\xi}\right\rangle:=\int_{\mathbb{S}^{2}} \boldsymbol{\xi} \otimes \boldsymbol{\xi} \mathrm{d} \nu_{t, x}^{\infty}(\boldsymbol{\xi}) .
$$

This is the only available framework which allows us to obtain (for any given initial datum) the long-time existence of solutions, which comply with basic physical principles such as the dissipation of energy (the existence of weak solutions for any initial datum, which violate the energy inequality, has been shown in [36]). The energy inequality implies a weak-strong uniqueness principle for measure-valued solutions as shown in [7]: A measure-valued solution coincides with the strong solution as soon as the strong solution exists.

While all these results concern the deterministic case, there is strong interest to study measurevalued solutions to the three-dimensional stochastic Euler equations (1.1) in order to grasp its long-term dynamics. The first result is the existence of martingale solutions in [29], where the equations of motion are understood in the measure-valued sense. These solutions are weak in the probabilistic sense, that is the underlying probability space as well as the driving Wiener process are not a priori given but become an integral part of the solution. Such a concept is common for stochastic evolutionary problems when uniqueness is not available. It is classical for finite dimensional problems and has also been applied to various stochastic partial differential equations, in particular in fluid mechanics (see, for instance, $[6,8,13,15,21])$. Unfortunately, the solutions constructed in [29] do only satisfy a form of energy estimate in expectation with an unspecified constant $C$ on the right-hand side, rather than an energy inequality as in the deterministic case. This is not enough to conclude with a weak-strong uniqueness principle which one should require for any reasonable notion of generalized solution, cf. [31].

The aim of this paper is to close this gap and to develop a concept of measure-valued martingale solutions to (1.1) which satisfy a suitable energy inequality. These solutions are called dissipative and our energy inequality can be described as follows: If $\mathcal{V}=\left(\nu_{t, x}, \nu_{t, x}^{\infty}, \lambda\right)$ is the generalised Young measure associated to the solution, then the kinetic energy (here $\mathscr{L}^{1}$ denotes the one-dimensional Lebesgue measure)

$$
E_{t}=\frac{1}{2} \int_{\mathbb{T}^{3}}\left\langle\nu_{t, x},|\boldsymbol{\xi}|^{2}\right\rangle \mathrm{d} x+\frac{1}{2} \lambda_{t}\left(\mathbb{T}^{3}\right), \quad \lambda=\lambda_{t} \otimes \mathscr{L}^{1}
$$

satisfies

$$
E_{t^{+}} \leq E_{s^{-}}+\frac{1}{2} \int_{s}^{t}\|\Phi\|_{L_{2}}^{2} \mathrm{~d} \tau+\int_{s}^{t} \int_{\mathbb{T}^{3}} \mathbf{u} \cdot \Phi \mathrm{d} x \mathrm{~d} W, \quad E_{0^{-}}=\frac{1}{2} \int_{\mathbb{T}^{3}}|\mathbf{u}(0)|^{2} \mathrm{~d} x,
$$

$\mathbb{P}$-a.s. for any $0 \leq s<t$, see Definition 3.1 for the precise formulation. In the deterministic case the energy is non-increasing and non-negative such that the left- and right-sided limits $E_{t^{-}}$and $E_{t^{+}}$exist for any $t$. In the stochastic case one has instead that the difference between the energy and a continuous function is nonincreasing and that both are pathwise bounded such that the same conclusion holds, see also Remark (3.2). Nevertheless, some care is required to implement this idea within the stochastic compactness method, see Sect.3.3. With the energy inequality just described at hand we are able to analyse the weak-strong uniqueness property of (1.1). In a pathwise approach we prove that a dissipative martingale solution agrees with the strong solution if both exist on the same probability space. This is reminiscent of the deterministic analysis in [7]. For this it is crucial that the energy inequality discussed above holds for any time $t$ in order to work with stopping times. A more realistic assumption is that the probability spaces, on which both solutions exit, are distinct. In this situation we prove that the probability laws of the weak and the strong solution coincide. This is based on the classical Yamada-Watanabe argument, where a product probability space is constructed. Thereby, the weak-strong uniqueness in law can be reduced to the pathwise weak-strong uniqueness already obtained. We face several difficulties due to the fact that 
(1.1) is infinite-dimensional and, in particular, due to the non-separability of the space of generalised Young measures.

The paper is organised as follows. In Sect. 2 we present some preliminary material. In particular, we introduce the set-up for generalised Young measure, present the concept of random distributions from [4] (in order to define progressive measurability for stochastic processes which are only equivalence classes in time) and prove an infinite dimensional Itô-formula which is appropriate for our purposes. Finally, we collect some known material on the stochastic Navier-Stokes equations. The latter will be needed to approximate the stochastic Euler equations. In Sect. 3 we introduce the concept of dissipative martingale solutions and prove their existence. As in [29] we approximate (1.1) by a sequence of NavierStokes equations with vanishing viscosity and use a refined stochastic compactness method (based on Jakubowski's extension of Skorokhod's representation theorem [26]). Section 4 is dedicated to weakstrong uniqueness.

\section{Mathematical Framework}

In this section we present various preliminaries on generalised Young measures, random variables and stochastic integration. Moreover, we collect some known material on the stochastic Navier-Stokes equations.

\subsection{Generalised Young Measures}

We denote by $\mathscr{M}$ the set of Radon measures, by $\mathscr{M}^{+}$the set of non-negative Radon measures and by $\mathscr{P}$ the set of probability measures. In our application there will be usually defined on a parabolic cylinder $Q_{T}=(0, T) \times \mathbb{T}^{3}$. We will only use the integrability index $p=2$. Also, without further mentioning it, we will exclusively deal with generalised Young measures generated by sequences of functions with values in $\mathbb{R}^{3}$. A generalised Young measure is defined as follows.

Definition 2.1. A quantity $\mathcal{V}=\left(\nu_{t, x}, \nu_{t, x}^{\infty}, \lambda\right)$ is called generalised Young measure provided

(a) $(t, x) \mapsto \nu_{t, x} \in L_{w^{*}}^{\infty}\left(Q_{T} ; \mathscr{P}\left(\mathbb{R}^{3}\right)\right)$ is a parametrised probability measure on $\mathbb{R}^{3}$;

(b) $\lambda \in \mathscr{M}^{+}\left(Q_{T}\right)$ is a non-negative Radon measure;

(c) $(t, x) \mapsto \nu_{t, x}^{\infty} \in L_{w^{*}}^{\infty}\left(Q_{T}, \lambda ; \mathscr{P}\left(\mathbb{S}^{2}\right)\right)$ is a parametrised probability measure on $\mathbb{S}^{2}$;

(d) We have $\int_{Q_{T}}\left\langle\nu_{t, x},|\boldsymbol{\xi}|^{2}\right\rangle \mathrm{d} x \mathrm{~d} t<\infty$.

We denote the space of all generalised Young measure by $Y_{2}\left(Q_{T}\right)$.

In particular, any Radon measure $\mu \in \mathscr{M}\left(Q_{T} ; \mathbb{R}^{3}\right)$ can be represented by a generalized Young measure by setting $\mathcal{V}=\left(\delta_{\mu^{a}(t, x)}, \frac{\mathrm{d} \mu^{s}}{\mathrm{~d}\left|\mu^{s}\right|},\left|\mu^{s}\right|\right)$, where $\mu=\mu^{a} \mathrm{~d} \mathscr{L}^{3}+\mu^{s}$ is the Radon-Nikodým decomposition of $\mu$. We consider now all continuous functions $f: Q_{T} \times \mathbb{R}^{3} \rightarrow \mathbb{R}$ such that the recession function

$$
f^{\infty}(t, x, \boldsymbol{\xi}):=\lim _{s \rightarrow \infty} \frac{f(t, x, s \boldsymbol{\xi})}{s^{2}}
$$

is well-defined and continuous on $\bar{Q}_{T} \times \mathbb{S}^{2}$ (which implies that $f$ grows at most quadratically in $\boldsymbol{\xi}$ ). We denote by $\mathcal{G}_{2}\left(Q_{T}\right)$ the space of all such functions. We say a sequence $\left\{\mathcal{V}^{n}\right\}=\left\{\left(\nu_{t, x}^{n}, \nu_{t, x}^{\infty, n}, \lambda^{n}\right)\right\}$ converges weakly* in $Y_{2}\left(Q_{T}\right)$ to some $\mathcal{V}=\left(\nu_{t, x}, \nu_{t, x}^{\infty}, \lambda\right) \in Y_{2}\left(Q_{T}\right)$ provided

$$
\begin{aligned}
\int_{Q_{T}} & \left\langle\nu_{t, x}^{n}, f(t, x, \boldsymbol{\xi})\right\rangle \mathrm{d} x \mathrm{~d} t+\int_{Q_{T}}\left\langle\nu_{t, x}^{\infty, n}, f^{\infty}(t, x, \boldsymbol{\xi})\right\rangle \mathrm{d} \lambda^{n} \\
& \rightarrow \int_{Q_{T}}\left\langle\nu_{t, x}, f(t, x, \boldsymbol{\xi})\right\rangle \mathrm{d} x \mathrm{~d} t+\int_{Q_{T}}\left\langle\nu_{t, x}^{\infty}, f^{\infty}(t, x, \boldsymbol{\xi})\right\rangle \mathrm{d} \lambda
\end{aligned}
$$


for all $f \in \mathcal{G}_{2}\left(Q_{T}\right)$. Here $\boldsymbol{\xi} \in \mathbb{R}^{3}$ denotes the corresponding dummy-variable. The space $\mathcal{G}_{2}\left(Q_{T}\right)$ is a separable Banach space together with the norm

$$
\|f\|_{\mathcal{G}_{2}\left(Q_{T}\right)}:=\sup _{(t, x) \in Q_{T}, \boldsymbol{\xi} \in B_{1}(0)}(1-|\boldsymbol{\xi}|)^{2}\left|f\left(t, x, \frac{\boldsymbol{\xi}}{1-|\boldsymbol{\xi}|}\right)\right|
$$

and $Y_{2}\left(Q_{T}\right)$ is a subspace of its dual. Consequently, $Y_{2}\left(Q_{T}\right)$ together with the weak* convergence introduced above is a quasi-Polish space.

A topological space $(X, \tau)$ is called quasi-Polish space if there is a countable family

$$
\left\{f_{n}: X \rightarrow[-1,1] ; n \in \mathbb{N}\right\}
$$

of continuous functions that separates points. In particular, separable Banach spaces endowed with the weak topology and dual spaces of separable Banach spaces are quasi-Polish spaces. Since we are interested in the long-time behaviour we also define

$$
Y_{2}^{\text {loc }}\left(Q_{\infty}\right)=\left\{\mathcal{V}: \mathcal{V} \in Y_{2}\left(Q_{T}\right) \forall T>0\right\} .
$$

Since the topology on $Y_{2}^{\text {loc }}\left(Q_{\infty}\right)$ is generated by the topologies on $Y_{2}\left(Q_{T}\right)$ in the sense that

$$
\mathcal{V}^{n} \rightarrow^{*} \mathcal{V} \quad \text { in } Y_{2}^{\text {loc }}\left(Q_{\infty}\right) \quad \Leftrightarrow \quad \mathcal{V}^{n} \rightarrow^{*} \mathcal{V} \quad \text { in } \quad Y_{2}\left(Q_{T}\right) \quad \forall T>0
$$

it is clear that $Y_{2}^{\mathrm{loc}}\left(Q_{\infty}\right)$ is a quasi-Polish space as well.

We can embed $L^{2}\left(Q_{T}\right)$ into $Y_{2}\left(Q_{T}\right)$ via the inclusion

$$
L^{2}\left(Q_{T}\right) \ni u \mapsto\left(\delta_{u(t, x)}, 0,0\right) \in Y_{2}\left(Q_{T}\right) .
$$

By the Alaoglu-Bourbaki theorem, for any $L>0$ there is a weak* compact subset $\mathcal{K}_{L}$ of $\mathcal{G}_{2}\left(Q_{T}\right)^{*}$ such that

$$
\left\{\left(\delta_{u(t, x)}, 0,0\right) \in Y_{2}\left(Q_{T}\right):\|u\|_{L^{2}\left(Q_{T}\right)} \leq L\right\} \subset \mathcal{K}_{L}
$$

Since $Y_{2}\left(Q_{T}\right)$ is weak ${ }^{*}$ closed in $\mathcal{G}_{2}\left(Q_{T}\right)^{*}$ we conclude that $\mathcal{K}_{L} \cap Y_{2}\left(Q_{T}\right)$ is weak* compact, where clearly

$$
\left\{\left(\delta_{u(t, x)}, 0,0\right) \in Y_{2}\left(Q_{T}\right):\|u\|_{L^{2}\left(Q_{T}\right)} \leq L\right\} \subset \mathcal{K}_{L} \cap Y_{2}\left(Q_{T}\right) .
$$

It is also useful to identify a generalised Young measure with a space-time distribution: For $\mathcal{V}=$ $\left(\nu_{t, x}, \nu_{t, x}^{\infty}, \lambda\right) \in Y_{2}\left(Q_{T}\right)$ we define

$$
\begin{aligned}
C_{c}^{\infty}\left(Q_{T} \times \mathbb{R}^{3}\right)^{2} \ni(\psi, \varphi) \mapsto & \int_{Q_{T}} \int_{\mathbb{R}^{3}} \psi(t, x, \boldsymbol{\xi}) \mathrm{d} \nu_{t, x}(\boldsymbol{\xi}) \mathrm{d} x \mathrm{~d} t \\
& +\int_{Q_{T}} \int_{\mathbb{R}^{3}} \varphi(t, x, \xi) \mathrm{d} \nu_{t, x}^{\infty}(\boldsymbol{\xi}) \mathrm{d} \lambda(t, x) .
\end{aligned}
$$

As we will study probability laws on $Y_{2}\left(Q_{T}\right)$ we need a $\sigma$-field. A suitable candidate is the $\sigma$-algebra generated by the functions $\left\{f_{n}\right\}$ from $(2.1)$, that is we set

$$
\mathscr{B}_{Y}:=\sigma\left(\bigcup_{n=1}^{\infty} \sigma\left(f_{n}\right)\right) \text {. }
$$

\subsection{Random Distributions}

Let $Q_{T}=(0, T) \times \mathbb{T}^{3}$. Let $\left(\Omega, \mathfrak{F},\left(\mathfrak{F}_{t}\right)_{t \geq 0}, \mathbb{P}\right)$ be a complete stochastic basis with a probability measure $\mathbb{P}$ on $(\Omega, \mathfrak{F})$ and a right-continuous filtration $\left(\mathfrak{F}_{t}\right)$. For a measurable space $(X, \mathcal{A})$ an $X$-valued random variable is a measurable mapping $\mathbf{U}:(\Omega, \mathfrak{F}) \rightarrow(X, \mathcal{A})$. We denote by $\sigma(\mathbf{U})$ the smallest $\sigma$-field with respect to which $\mathbf{U}$ is measurable, that is

$$
\sigma(\mathbf{U}):=\{\{\omega \in \Omega ; \mathbf{U}(\omega) \in A\} ; A \in \mathcal{A}\} .
$$

In order to deal with oscillations and concentrations in the convective term of approximate solutions to the stochastic Euler equations we have to deal with generalised Young measures (as introduced in the 
previous subsection) and hence we need to study mappings $\mathbf{U}: \Omega \rightarrow Y_{2}\left(Q_{T}\right)$. Such an object is not a stochastic process in the classical sense as it is only defined a.e. in time. Consequently, it becomes ambiguous to speak about progressive measurability. To overcome such problems the concept of random distributions has been introduced in [4, Chap. 2.2] to which we refer to for more details.

Definition 2.2. Let $(\Omega, \mathfrak{F}, \mathbb{P})$ be a complete probability space and $N \in \mathbb{N}$. A mapping

$$
\mathbf{U}: \Omega \rightarrow\left(C_{c}^{\infty}\left(Q_{T} ; \mathbb{R}^{N}\right)\right)^{\prime}
$$

is called random distribution if $\langle\mathbf{U}, \varphi\rangle: \Omega \rightarrow \mathbb{R}$ is a measurable function for any $\varphi \in C_{c}^{\infty}\left(Q_{T} ; \mathbb{R}^{N}\right)$.

In order to introduce a concept of progressive measurability we consider the $\sigma$-field of all progressively measurable sets in $\Omega \times[0, T]$ associated to the filtration $\left(\mathfrak{F}_{t}\right)_{t \geq 0}$. To be more precise, $A \subset \Omega \times[0, T]$ belongs to the progressively measurable $\sigma$-field provided the stochastic process $(\omega, t) \mapsto \mathbb{I}_{A}(\omega, t)$ is $\left(\mathfrak{F}_{t}\right)$ progressively measurable. We denote by $L_{\text {prog }}^{1}(\Omega \times[0, T])$ the Lebesgue space of functions that are measurable with respect to the $\sigma$-field of $\left(\mathfrak{F}_{t}\right)$-progressively measurable sets in $\Omega \times[0, T]$ and we denote by $\mu_{\text {prog }}$ the measure $\left.\mathbb{P} \otimes \mathscr{L}^{1}\right|_{[0, T]}$ restricted to the progressively measurable $\sigma$-field.

Definition 2.3. Let $\mathbf{U}$ be a random distribution in the sense of Definition 2.2.

(a) We say that $\mathbf{U}$ is adapted to $\left(\mathfrak{F}_{t}\right)$ if $\langle\mathbf{U}, \boldsymbol{\varphi}\rangle$ is $\left(\mathfrak{F}_{t}\right)$-measurable for any $\varphi \in C_{c}^{\infty}\left(Q_{t} ; \mathbb{R}^{N}\right)$.

(b) We say that $\mathbf{U}$ is $\left(\mathfrak{F}_{t}\right)$-progressively measurable if $\langle\mathbf{U}, \boldsymbol{\varphi}\rangle \in L_{\operatorname{prog}}^{1}(\Omega \times[0, T])$ for any $\boldsymbol{\varphi} \in C_{c}^{\infty}\left(Q_{T} ; \mathbb{R}^{N}\right)$.

The above concept is convenient when dealing with general distributions. It coincides with the standard concept of progressive measurability as long as the distribution defines a stochastic process, see [4, Chapter 2, Lemma 2.2.18]. Also, if a random distribution is $\left(\mathfrak{F}_{t}\right)$-adapted, there is a modification which is $\left(\mathfrak{F}_{t}\right)$ progressively measurable, cf. [4, Chapter 2, Lemma 2.2.18], as in the classical situation. The family of $\sigma$-fields $\left(\sigma_{t}[\mathbf{U}]\right)_{t \geq 0}$ given as

$$
\sigma_{t}[\mathbf{U}]:=\bigcap_{s>t} \sigma\left(\bigcup_{\varphi \in C_{c}^{\infty}\left(Q_{s} ; \mathbb{R}^{N}\right)}\{\langle\mathbf{U}, \varphi\rangle<1\} \cup\{N \in \mathfrak{F}, \mathbb{P}(N)=0\}\right)
$$

is called history of $\mathbf{U}$. Clearly, any random distribution is adapted to its history.

\subsection{Stochastic Analysis}

Let $\left(\Omega, \mathfrak{F},\left(\mathfrak{F}_{t}\right)_{t \geq 0}, \mathbb{P}\right)$ be a complete stochastic basis with a probability measure $\mathbb{P}$ on $(\Omega, \mathfrak{F})$ and a rightcontinuous filtration $\left(\mathfrak{F}_{t}\right)$. We refer the reader to [12] for more details on the following elements of stochastic calculus in infinite dimensions. Let $\mathfrak{U}$ be a separable Hilbert space and let $\left(\mathbf{e}_{k}\right)_{k \in \mathbb{N}}$ be an orthonormal basis of $\mathfrak{U}$. We denote by $L_{2}\left(\mathfrak{U}, L^{2}\left(\mathbb{T}^{3}\right)\right)$ the set of Hilbert-Schmidt operators from $\mathfrak{U}$ to $L^{2}\left(\mathbb{T}^{3}\right)$. Throughout the paper we consider a cylindrical Wiener process $W=\left(W_{t}\right)_{t \geq 0}$ which has the form

$$
W(\sigma)=\sum_{k \in \mathbb{N}} \beta_{k}(\sigma) \mathbf{e}_{k}
$$

with a sequence $\left(\beta_{k}\right)$ of independent real valued Brownian motions on $\left(\Omega, \mathfrak{F},\left(\mathfrak{F}_{t}\right)_{t \geq 0}, \mathbb{P}\right)$. The stochastic integral

$$
\int_{0}^{t} \psi \mathrm{d} W, \quad \psi \in L^{2}\left(\Omega, \mathfrak{F}, \mathbb{P} ; L^{2}\left(0, T ; L_{2}\left(\mathfrak{U}, L^{2}\left(\mathbb{T}^{3}\right)\right)\right),\right.
$$

where $\psi$ is $\left(\mathfrak{F}_{t}\right)$-progressively measurable, defines a $\mathbb{P}$-almost surely continuous $L^{2}\left(\mathbb{T}^{3}\right)$ valued $\left(\mathfrak{F}_{t}\right)$ martingale. Moreover, we can multiply with test-functions since

$$
\left\langle\int_{0}^{t} \psi \mathrm{d} W, \boldsymbol{\varphi}\right\rangle_{L^{2}\left(\mathbb{T}^{3}\right)}=\sum_{k=1}^{\infty} \int_{0}^{t}\left\langle\psi\left(\mathbf{e}_{k}\right), \boldsymbol{\varphi}\right\rangle_{L^{2}\left(\mathbb{T}^{3}\right)} \mathrm{d} \beta_{k}, \quad \boldsymbol{\varphi} \in L^{2}\left(\mathbb{T}^{3}\right),
$$


is well-defined (the series converges in $L^{2}(\Omega, \mathfrak{F}, \mathbb{P} ; C[0, T])$ ).

Define further $\mathfrak{U}_{0} \supset \mathfrak{U}$ as

$$
\mathfrak{U}_{0}:=\left\{\mathbf{e}=\sum_{k} \alpha_{k} \mathbf{e}_{k}: \sum_{k} \frac{\alpha_{k}^{2}}{k^{2}}<\infty\right\},
$$

thus the embedding $\mathfrak{U} \hookrightarrow \mathfrak{U}_{0}$ is Hilbert-Schmidt and trajectories of $W$ are $\mathbb{P}$-a.s. continuous with values in $\mathfrak{U}_{0}$.

The following infinite dimensional Itô-formula is a variant of [3, Lemma 3.1].

Lemma 2.4. Let $\left(\Omega, \mathfrak{F},\left(\mathfrak{F}_{t}\right)_{t \geq 0}, \mathbb{P}\right)$ be a stochastic basis and let $W$ be a cylindrical $\left(\mathfrak{F}_{t}\right)$-Wiener process. Let $\mathbf{w}^{1}, \mathbf{w}^{2}$ be $\left(\mathfrak{F}_{t}\right)$-progressively measurable satisfying $\mathbf{w}^{1} \in C_{w}\left([0, T] ; L_{\mathrm{div}}^{2}\left(\mathbb{T}^{3}\right)\right), \mathbf{w}^{2} \in C\left([0, T] ; L_{\mathrm{div}}^{2}\left(\mathbb{T}^{3}\right)\right)$ and $\mathbf{w}^{2} \in L^{1}\left(0, T ; C^{1}\left(\mathbb{T}^{3}\right)\right)$ a.s. such that

$$
\mathbf{w}^{1}, \mathbf{w}^{2} \in L_{w^{*}}^{2}\left(\Omega ; L^{\infty}\left(0, T ; L^{2}\left(\mathbb{T}^{3}\right)\right)\right) .
$$

Suppose that there are

$$
\begin{gathered}
\lambda_{t} \in L_{w^{*}}^{1}\left(\Omega ; L_{w^{*}}^{\infty}\left(0, T ; \mathscr{M}^{+}\left(\mathbb{T}^{3}\right)\right)\right), \quad \Phi^{1} \in L^{2}\left(\Omega ; L^{2}\left(0, T ; L_{2}\left(\mathfrak{U} ; L^{2}\left(\mathbb{T}^{3}\right)\right)\right),\right. \\
\mathbf{H}^{1} \in L_{w^{*}}^{1}\left(\Omega ; L^{\infty}\left(0, T ; L^{1}\left(\mathbb{T}^{3}\right)\right)\right),
\end{gathered}
$$

as well as a random distribution $\mathbf{G}^{1}$ such that $\mathbf{G}^{1} \in L^{\infty}\left(Q_{T}, \lambda_{t} \otimes \mathscr{L}^{1}\right) \mathbb{P}$-a.s. and

$$
\mathbb{E}\left[\inf _{\lambda_{t} \otimes \mathcal{L}^{1}(\mathscr{N})=0}\left\|\mathbf{G}^{1}\right\|_{L^{\infty}\left(Q_{T} \backslash \mathscr{N}\right)}\right]<\infty .
$$

We further assume that $\lambda_{t}, \Phi^{1}, \mathbf{H}^{1}$ and $\mathbf{G}^{1}$ are progressively $\left(\mathfrak{F}_{t}\right)$-measurable and that

$$
\begin{aligned}
\int_{\mathbb{T}^{3}} \mathbf{w}^{1}(t) \cdot \boldsymbol{\varphi} \mathrm{d} x= & \int_{\mathbb{T}^{3}} \mathbf{w}^{1}(0) \cdot \boldsymbol{\varphi} \mathrm{d} x+\int_{0}^{t} \int_{\mathbb{T}^{3}} \mathbf{H}^{1}: \nabla \boldsymbol{\varphi} \mathrm{d} x \mathrm{~d} \sigma \\
& +\int_{0}^{t} \int_{\mathbb{T}^{3}} \mathbf{G}^{1}: \nabla \boldsymbol{\varphi} \mathrm{d} \lambda_{\sigma} \mathrm{d} \sigma+\int_{\mathbb{T}^{3}} \boldsymbol{\varphi} \cdot \int_{0}^{t} \Phi^{1} \mathrm{~d} W \mathrm{~d} x
\end{aligned}
$$

for all $\varphi \in C_{\mathrm{div}}^{\infty}\left(\mathbb{T}^{3}\right)$.

Suppose further that there are

$$
\mathbf{h}^{2} \in L_{w^{*}}^{1}\left(\Omega ; L^{\infty}(Q)\right), \quad \Phi^{2} \in L^{2}\left(\Omega ; L^{2}\left(0, T ; L_{2}\left(\mathfrak{U} ; L^{2}\left(\mathbb{T}^{3}\right)\right)\right),\right.
$$

$\left(\mathfrak{F}_{t}\right)$-progressively measurable such that

$$
\begin{aligned}
\int_{\mathbb{T}^{3}} \mathbf{w}^{2}(t) \cdot \boldsymbol{\varphi} \mathrm{d} x= & \int_{\mathbb{T}^{3}} \mathbf{w}^{2}(0) \cdot \boldsymbol{\varphi} \mathrm{d} x+\int_{0}^{t} \int_{\mathbb{T}^{3}} \mathbf{h}^{2} \cdot \boldsymbol{\varphi} \mathrm{d} x \mathrm{~d} \sigma \\
& +\int_{\mathbb{T}^{3}} \boldsymbol{\varphi} \cdot \int_{0}^{t} \Phi^{2} \mathrm{~d} W \mathrm{~d} x
\end{aligned}
$$

for all $\varphi \in C_{\operatorname{div}}^{\infty}\left(\mathbb{T}^{3}\right)$. Then we have for all $t \geq 0 \mathbb{P}$-a.s.

$$
\begin{aligned}
\int_{\mathbb{T}^{3}} \mathbf{w}^{1}(t) \cdot \mathbf{w}^{2}(t) \mathrm{d} x= & \int_{\mathbb{T}^{3}} \mathbf{w}^{1}(0) \cdot \mathbf{w}^{2}(0) \mathrm{d} x+\int_{0}^{t} \int_{\mathbb{T}^{3}} \mathbf{H}^{1}: \nabla \mathbf{w}^{2} \mathrm{~d} x \mathrm{~d} \sigma \\
& +\int_{0}^{t} \int_{\mathbb{T}^{3}} \mathbf{G}^{1}: \nabla \mathbf{w}^{2} \mathrm{~d} \lambda_{\sigma} \mathrm{d} \sigma+\int_{\mathbb{T}^{3}} \mathbf{w}^{2} \cdot \int_{0}^{t} \Phi^{1} \mathrm{~d} W \mathrm{~d} x \\
& +\int_{0}^{t} \int_{\mathbb{T}^{3}} \mathbf{h}^{2} \cdot \mathbf{w}^{1} \mathrm{~d} x \mathrm{~d} \sigma+\int_{\mathbb{T}^{3}} \mathbf{w}^{1} \cdot \int_{0}^{t} \Phi^{2} \mathrm{~d} W \mathrm{~d} x \\
& +\sum_{k \geq 1} \int_{0}^{t} \int_{\mathbb{T}^{3}} \Phi^{1} \mathbf{e}_{k} \cdot \Phi^{2} \mathbf{e}_{k} \mathrm{~d} x \mathrm{~d} t .
\end{aligned}
$$


Proof. In order to justify the application of Itô's formula to the process $t \mapsto \int_{\mathbb{T}^{3}} \mathbf{w}^{1}(t) \cdot \mathbf{w}^{2}(t) \mathrm{d} x$ we have to perform some regularisations in equation (2.9) using mollification in space with parameter $\varrho>0$. For $\varphi \in L_{\mathrm{div}}^{2}\left(\mathbb{T}^{3}\right)$ we have $\varphi_{\varrho} \in C_{\mathrm{div}}^{\infty}\left(\mathbb{T}^{3}\right)$ and

$$
\begin{aligned}
& \left\|\varphi_{\varrho}\right\|_{W_{x}^{k, p}} \leq c(\varrho)\|\varphi\|_{L_{x}^{2}} \quad \forall k \in \mathbb{N}_{0}, p \in[1, \infty], \\
& \left\|\varphi_{\varrho}\right\|_{W_{x}^{k, p}} \leq\|\varphi\|_{W_{x}^{k, 2}} \quad \forall k \in \mathbb{N}_{0}, p \in[1, \infty],
\end{aligned}
$$

provided $\varphi \in L^{p}\left(\mathbb{T}^{3}\right)$ or $\varphi \in W^{k, p}\left(\mathbb{T}^{3}\right)$ respectively. Moreover,

$$
\begin{array}{ll}
\boldsymbol{\varphi}_{\varrho} \rightarrow \boldsymbol{\varphi} & \text { in } \quad W^{k, p}\left(\mathbb{T}^{3}\right) \quad \forall k \in \mathbb{N}_{0}, p \in[1, \infty), \\
\boldsymbol{\varphi}_{\varrho} \rightarrow \boldsymbol{\varphi} & \text { in } \quad C^{k}\left(\mathbb{T}^{3}\right) \quad \forall k \in \mathbb{N}_{0},
\end{array}
$$

as $\varrho \rightarrow 0$ provided $\varphi \in W^{k, p}\left(\mathbb{T}^{3}\right)$ or $C^{k}\left(\mathbb{T}^{3}\right)$ respectively. Finally, the operator $(\cdot)_{\varrho}$ commutes with derivatives. Inserting $\varphi_{\varrho}$ in $(2.9)$ yields

$$
\begin{aligned}
\int_{\mathbb{T}^{3}} \mathbf{w}_{\varrho}^{1}(t) \cdot \boldsymbol{\varphi} \mathrm{d} x= & \int_{\mathbb{T}^{3}} \mathbf{w}_{\varrho}^{1}(0) \cdot \boldsymbol{\varphi} \mathrm{d} x+\int_{0}^{t} \int_{\mathbb{T}^{3}} \mathbf{H}^{1}: \nabla(\boldsymbol{\varphi})_{\varrho} \mathrm{d} x \mathrm{~d} \sigma \\
& +\int_{0}^{t} \int_{\mathbb{T}^{3}} \mathbf{G}^{1}: \nabla(\boldsymbol{\varphi})_{\varrho} \mathrm{d} \lambda_{\sigma} \mathrm{d} \sigma+\int_{\mathbb{T}^{3}} \boldsymbol{\varphi} \cdot \int_{0}^{t} \Phi_{\varrho}^{1} \mathrm{~d} W \mathrm{~d} x,
\end{aligned}
$$

where $\Phi_{\varrho}^{1}$ is given by $\Phi_{\varrho}^{1} \mathbf{e}_{k}=\left(\Phi^{1} \mathbf{e}_{k}\right)_{\varrho}$ for $k \in \mathbb{N}$. Using (2.12) we have for fixed $\varrho>0$

$$
\begin{aligned}
\left|\int_{0}^{t} \int_{\mathbb{T}^{3}} \mathbf{H}^{1}: \nabla(\boldsymbol{\varphi}) \varrho \mathrm{d} x \mathrm{~d} \sigma\right| & \leq \sup _{0 \leq t \leq T} \int_{\mathbb{T}^{3}}\left|\mathbf{H}^{1}\right| \mathrm{d} x \int_{0}^{T}\|\nabla(\boldsymbol{\varphi}) \varrho\|_{L_{x}^{\infty}} \mathrm{d} \sigma \\
& \leq c(\varrho) \sup _{0 \leq t \leq T} \int_{\mathbb{T}^{3}}\left|\mathbf{H}^{1}\right| \mathrm{d} x \int_{0}^{T}\|\boldsymbol{\varphi}\|_{L_{x}^{2}} \mathrm{~d} \sigma
\end{aligned}
$$

$\mathbb{P}$-a.s. as well as

$$
\begin{aligned}
\left|\int_{0}^{t} \int_{\mathbb{T}^{3}} \mathbf{G}^{1}: \nabla(\boldsymbol{\varphi})_{\varrho} \mathrm{d} \lambda_{\sigma} \mathrm{d} \sigma\right| & \leq \sup _{Q_{T}}\left|\mathbf{G}^{1}\right| \int_{0}^{T} \int_{\mathbb{T}^{3}}|\nabla(\boldsymbol{\varphi}) \varrho| \mathrm{d} \lambda_{\sigma} \mathrm{d} \sigma \\
& \leq \sup _{Q_{T}}\left|\mathbf{G}^{1}\right| \sup _{0 \leq \sigma \leq T} \lambda_{\sigma}\left(\mathbb{T}^{3}\right) \int_{0}^{T}\left\|\nabla(\boldsymbol{\varphi})_{\varrho}\right\|_{L_{x}^{\infty}} \mathrm{d} \sigma \\
& \leq c(\varrho) \sup _{Q_{T}}\left|\mathbf{G}^{1}\right| \sup _{0 \leq \sigma \leq T} \lambda_{\sigma}\left(\mathbb{T}^{3}\right) \int_{0}^{T}\|\varphi\|_{L_{x}^{2}} \mathrm{~d} \sigma .
\end{aligned}
$$

Hence the deterministic parts in the equation for $\mathbf{w}_{\varrho}^{1}$ are functionals on $L^{2}$. Consequently, we can apply Itô's formula on the Hilbert space $L_{\mathrm{div}}^{2}\left(\mathbb{T}^{3}\right)$ (see [12, Thm. 4.17]) to the process $t \mapsto \int_{\mathbb{T}^{3}} \mathbf{w}_{\varrho}^{1}(t) \cdot \mathbf{w}^{2}(t) \mathrm{d} x$ to obtain

$$
\begin{aligned}
\int_{\mathbb{T}^{3}} \mathbf{w}_{\varrho}^{1}(t) \cdot \mathbf{w}^{2}(t) \mathrm{d} x= & \int_{\mathbb{T}^{3}} \mathbf{w}_{\varrho}^{1}(0) \cdot \mathbf{w}^{2}(0) \mathrm{d} x+\int_{0}^{t} \int_{\mathbb{T}^{3}} \mathbf{H}^{1}:\left(\nabla \mathbf{w}^{2}\right)_{\varrho} \mathrm{d} x \mathrm{~d} \sigma \\
& +\int_{0}^{t} \int_{\mathbb{T}^{3}} \mathbf{G}^{1}:\left(\nabla \mathbf{w}^{2}\right)_{\varrho} \mathrm{d} \lambda_{\sigma} \mathrm{d} \sigma+\int_{\mathbb{T}^{3}} \int_{0}^{t} \mathbf{w}^{2} \cdot \Phi_{\varrho}^{1} \mathrm{~d} W \mathrm{~d} x \\
& +\int_{0}^{t} \int_{\mathbb{T}^{3}} \mathbf{h}^{2} \cdot \mathbf{w}_{\varrho}^{1} \mathrm{~d} x \mathrm{~d} \sigma+\int_{\mathbb{T}^{3}} \int_{0}^{t} \mathbf{w}_{\varrho}^{1} \cdot \Phi^{2} \mathrm{~d} W \mathrm{~d} x \\
& +\sum_{k \geq 1} \int_{0}^{t} \int_{\mathbb{T}^{3}} \Phi_{\varrho}^{1} \mathbf{e}_{k} \Phi^{2} \mathbf{e}_{k} \mathrm{~d} x \mathrm{~d} t .
\end{aligned}
$$

Passing to the limit $\varrho \rightarrow 0$ and using (2.13) together with the assumptions on $\mathbf{w}^{1}$ and $\mathbf{w}^{2}$ we see that all terms converge to their corresponding counterparts and (2.11) follows.

We conclude this section with a finite dimensional version of [4, Chapter 2, Theorem 2.9.1]. The proof of which follows along the same line (in fact, it is even simpler). 
Proposition 2.5. Let $U$ be a random distribution such that $U \in L_{\text {loc }}^{1}([0, \infty)) \mathbb{P}$-a.s. Suppose that there is a bounded continuous function $b$ and a collection of random distributions $\mathbb{G}=\left(G_{k}\right)_{k=1}^{\infty}$ such that $\mathbb{P}$-a.s.

$$
\sum_{k=1}^{\infty}\left|G_{k}\right|^{2} \in L_{\mathrm{loc}}^{1}([0, \infty)) .
$$

Let $U_{0}$ be an $\mathfrak{F}_{0}$-measurable random variable and let $W=\left(W_{k}\right)_{k=1}^{\infty}$ be a collection of real-valued independent Brownian motions. Suppose that the filtration

$$
\mathfrak{F}_{t}=\sigma\left(\sigma\left(U_{0}, \mathbf{r}_{t} U, \mathbf{r}_{t} W, \mathbf{r}_{t} \mathbb{G}\right)\right), t \geq 0,
$$

is non-anticipative with respect to $W$. Let $\tilde{U}_{0}$ be another random distribution and $\tilde{W}=\left(\tilde{W}_{k}\right)_{k=1}^{\infty}$ another stochastic process and random distributions $\tilde{\mathbb{G}}=\left(\tilde{G}_{k}\right)_{k=1}^{\infty}$, such their joint laws coincide, namely,

$$
\mathcal{L}\left[U_{0}, U, W, \mathbb{G}\right]=\mathcal{L}\left[\tilde{U}_{0}, \tilde{U}, \tilde{W}, \tilde{\mathbb{G}}\right] \text { or }\left[U_{0}, U, W, \mathbb{G}\right] \stackrel{d}{\sim}[\tilde{U} 0, \tilde{U}, \tilde{W}, \tilde{\mathbb{G}}] .
$$

Then $\tilde{W}$ is a collection of real-valued independent Wiener processes, the filtration

$$
\tilde{\mathfrak{F}}_{t}=\sigma\left(\sigma\left(\tilde{U}_{0}, \mathbf{r}_{t} \tilde{U}, \mathbf{r}_{t} \tilde{W}, \mathbf{r}_{t} \tilde{\mathbb{G}}\right)\right), t \geq 0,
$$

is non-anticipative with respect to $\tilde{W}, \tilde{U}_{0}$ is $\tilde{\mathfrak{F}}_{0}$-measurable, and

$$
\begin{aligned}
& \mathcal{L}\left[\int_{0}^{\infty}\left[\partial_{t} \psi U+b(U) \psi\right] \mathrm{d} t+\int_{0}^{\infty} \sum_{k=1}^{\infty} \psi G_{k} \mathrm{~d} W_{k}+\psi(0) U_{0}\right] \\
& \quad=\mathcal{L}\left[\int_{0}^{\infty}\left[\partial_{t} \psi \tilde{U}+b(\tilde{U}) \psi\right] \mathrm{d} t+\int_{0}^{\infty} \sum_{k=1}^{\infty} \psi \tilde{G}_{k} \mathrm{~d} \tilde{W}_{k}+\psi(0) \tilde{U}_{0}\right]
\end{aligned}
$$

for any deterministic $\psi \in C_{c}^{\infty}([0, \infty))$.

\subsection{Stochastic Navier-Stokes Equations}

The Euler equations are linked via a vanishing viscosity limit to the Navier-Stokes equations. The stochastic Navier-Stokes equations with viscosity $\mu>0$ read as

$$
\begin{cases}\operatorname{du}=\mu \Delta \mathbf{u} \mathrm{d} t-(\nabla \mathbf{u}) \mathbf{u} \mathrm{d} t-\nabla \pi \mathrm{d} t+\Phi \mathrm{d} W & \text { in } Q, \\ \operatorname{div} \mathbf{u}=0 & \text { in } Q,\end{cases}
$$

Here $W$ is a cylindrical Wiener process as introduced in the previous subsection. In the following we give a rigorous definition of a solution to (2.15).

Definition 2.6 [Solution]. Let $\Lambda$ be a Borel probability measure on $L_{\text {div }}^{2}\left(\mathbb{T}^{3}\right)$ and let $\Phi \in L_{2}\left(\mathfrak{U} ; L^{2}\left(\mathbb{T}^{3}\right)\right)$. Then

$$
\left(\left(\Omega, \mathfrak{F},\left(\mathfrak{F}_{t}\right), \mathbb{P}\right), \mathbf{u}, W\right)
$$

is called a finite energy weak martingale solution to (2.15) with the initial data $\Lambda$ provided

(a) $\left(\Omega, \mathfrak{F},\left(\mathfrak{F}_{t}\right), \mathbb{P}\right)$ is a stochastic basis with a complete right-continuous filtration;

(b) $W$ is an $\left(\mathfrak{F}_{t}\right)$-cylindrical Wiener process;

(c) The velocity field $\mathbf{u}$ is $\left(\mathfrak{F}_{t}\right)$-adapted and satisfies $\mathbb{P}$-a.s.

$$
\mathbf{u} \in C_{\mathrm{loc}}\left([0, \infty), W_{\operatorname{div}}^{-2,2}\left(\mathbb{T}^{3}\right)\right) \cap C_{w, \mathrm{loc}}\left([0, \infty) ; L_{\operatorname{div}}^{2}\left(\mathbb{T}^{3}\right)\right) \cap L_{\mathrm{loc}}^{2}\left(0, \infty ; W_{\operatorname{div}}^{1,2}\left(\mathbb{T}^{3}\right)\right) ;
$$

(d) $\Lambda=\mathbb{P} \circ(\mathbf{u}(0))^{-1}$; 
(e) For all $\varphi \in C_{\operatorname{div}}^{\infty}\left(\mathbb{T}^{3}\right)$ and all $t \geq 0$ there holds $\mathbb{P}$-a.s.

$$
\begin{aligned}
\int_{\mathbb{T}^{3}} \mathbf{u}(t) \cdot \boldsymbol{\varphi} \mathrm{d} x= & \int_{\mathbb{T}^{3}} \mathbf{u}(0) \cdot \boldsymbol{\varphi} \mathrm{d} x+\int_{0}^{t} \int_{\mathbb{T}^{3}} \mathbf{u} \otimes \mathbf{u}: \nabla \boldsymbol{\varphi} \mathrm{d} x \mathrm{~d} s \\
& -\mu \int_{0}^{t} \int_{\mathbb{T}^{3}} \nabla \mathbf{u}: \nabla \boldsymbol{\varphi} \mathrm{d} x \mathrm{~d} s+\int_{0}^{t} \int_{\mathbb{T}^{3}} \boldsymbol{\varphi} \cdot \Phi \mathrm{d} x \mathrm{~d} W ;
\end{aligned}
$$

(f) The energy inequality holds in the sense that

$$
\begin{aligned}
E_{t} & +\mu \int_{s}^{t} \int_{\mathbb{T}^{3}}|\nabla \mathbf{u}|^{2} \mathrm{~d} x \mathrm{~d} \sigma \\
& \leq E_{s}+\frac{1}{2} \int_{s}^{t}\|\Phi\|_{L_{2}\left(\left(\mathfrak{U}, L^{2}\left(\mathbb{T}^{3}\right)\right)\right)}^{2} \mathrm{~d} \sigma+\int_{s}^{t} \int_{\mathbb{T}^{3}} \mathbf{u} \cdot \Phi \mathrm{d} W
\end{aligned}
$$

$\mathbb{P}$-a.s. for a.a. $s \geq 0$ (including $s=0$ ) and all $t \geq s$, where $E_{t}=\frac{1}{2} \int_{\mathbb{T}^{3}}|\mathbf{u}(t)|^{2} \mathrm{~d} x$.

Definition 2.6 is standard in the theory of stochastic Navier-Stokes equations and can be found in a similar form, for instance, in [21] or [22]. The energy inequality in (f) is in the spirit of [22], but slightly differs and is reminiscent of the recent result for compressible fluids from [4]. Formerly, one can easily derive it by applying Itô's formula to the functional $t \mapsto \frac{1}{2} \int_{\mathbb{T}^{3}}|\mathbf{u}(t)|^{2} \mathrm{~d} x$. It can be made rigorous on the Galerkin level (even with equality). Consequently, the following existence theorem for (2.15) holds.

Theorem 2.7. Assume that we have

$$
\int_{L_{\mathrm{div}}^{2}\left(\mathbb{T}^{3}\right)}\|\mathbf{w}\|_{L_{x}^{2}}^{p} \mathrm{~d} \Lambda(\mathbf{w})<\infty
$$

for some $p>2$. Then there is a martingale solution to (2.15) in the sense of Definition 2.6.

\section{Dissipative Solutions}

In this section we formalise the concept of dissipative solutions to the stochastic Euler equations and prove their existence. The equations of interest read as

$$
\begin{cases}\mathrm{d} \mathbf{u}=-(\nabla \mathbf{u}) \mathbf{u} \mathrm{d} t-\nabla \pi \mathrm{d} t+\Phi \mathrm{d} W & \text { in } Q, \\ \operatorname{div} \mathbf{u}=0 & \text { in } Q,\end{cases}
$$

Here $W$ is a cylindrical Wiener process as introduced in Sect.2.3. Given an initial law $\Lambda$ on $L_{\text {div }}^{2}\left(\mathbb{T}^{3}\right)$ a martingale solution to $(3.1)$ consists of a probability space $\left(\Omega, \mathfrak{F},\left(\mathfrak{F}_{t}\right), \mathbb{P}\right)$, an $\left(\mathfrak{F}_{t}\right)$-cylindrical Wiener process and the random variables $(\mathbf{u}, \mathcal{V})$. The law $\mathcal{L}[\mathbf{u}(0), \mathbf{u}, \mathcal{V}, W]$ of $[\mathbf{u}(0), \mathbf{u}, \mathcal{V}, W]$ is a measure on the path space

$$
\begin{aligned}
\mathcal{X}:= & L_{\operatorname{div}}^{2}\left(\mathbb{T}^{3}\right) \times C_{\mathrm{loc}}\left([0, \infty) ; W_{\mathrm{div}}^{-4,2}\left(\mathbb{T}^{3}\right)\right) \cap C_{w, l o c}\left([0, \infty) ; L_{\operatorname{div}}^{2}\left(\mathbb{T}^{3}\right)\right) \times Y_{2}^{\mathrm{loc}}\left(Q_{\infty}\right) \\
& \times C_{\mathrm{loc}}\left([0, \infty), \mathfrak{U}_{0}\right) .
\end{aligned}
$$

It is equipped with the $\sigma$-field

$$
\begin{aligned}
\mathscr{B}_{\mathcal{X}} & :=\mathscr{B}\left(L_{\mathrm{d}}^{2}\left(\mathbb{T}^{3}\right)\right) \otimes \mathscr{B}_{\mathbf{u}}^{\mathrm{loc}} \otimes \mathscr{B}_{Y}^{\mathrm{loc}} \otimes \mathscr{B}\left(C_{\mathrm{loc}}\left([0, \infty), \mathfrak{U}_{0}\right)\right), \\
\mathscr{B}_{\mathbf{u}}^{\mathrm{loc}} & :=\sigma\left(\mathscr { B } \left(C_{\mathrm{loc}}\left([0, \infty) ; W_{\mathrm{div}}^{-4,2}\left(\mathbb{T}^{3}\right)\right) \cap \mathscr{B}_{\infty}\left(C_{w, \mathrm{loc}}\left([0, \infty) ; L_{\mathrm{d}}^{2}\left(\mathbb{T}^{3}\right)\right)\right),\right.\right.
\end{aligned}
$$

where $\mathscr{B}_{Y}^{\text {loc }}$ is defined in accordance with (2.5). For a Polish space $\mathscr{Y}$ we denote by $\mathscr{B}(\mathscr{Y})$ its Borel $\sigma$-field and for a Banach space $X$ we denote by $\mathscr{B}_{\infty}\left(C_{w, l o c}([0, \infty) ; X)\right)$ the $\sigma$-field generated by the mappings

$$
C_{w, l o c}([0, \infty) ; X) \rightarrow X, \quad h \mapsto h(s), \quad s \geq 0 .
$$


Definition 3.1 (Dissipative Solution). Let $\Lambda$ be a Borel probability measure on $L_{\text {div }}^{2}\left(\mathbb{T}^{3}\right)$ and let $\Phi \in$ $L_{2}\left(\mathfrak{U} ; L^{2}\left(\mathbb{T}^{3}\right)\right)$. Then

$$
\left(\left(\Omega, \mathfrak{F},\left(\mathfrak{F}_{t}\right), \mathbb{P}\right), \mathbf{u}, \mathcal{V}, W\right)
$$

is called a dissipative martingale solution to (3.1) with the initial data $\Lambda$ provided

(a) $\left(\Omega, \mathfrak{F},\left(\mathfrak{F}_{t}\right), \mathbb{P}\right)$ is a stochastic basis with a complete right-continuous filtration;

(b) $W$ is an $\left(\mathfrak{F}_{t}\right)$-cylindrical Wiener process;

(c) The velocity field $\mathbf{u}$ is $\left(\mathfrak{F}_{t}\right)$-adapted and satisfies $\mathbb{P}$-a.s.

$$
\mathbf{u} \in C_{\mathrm{loc}}\left([0, \infty), W_{\mathrm{div}}^{-4,2}\left(\mathbb{T}^{3}\right)\right) \cap C_{w, l o c}\left(0, \infty ; L_{\operatorname{div}}^{2}\left(\mathbb{T}^{3}\right)\right) ;
$$

(d) $\mathcal{V}=\left(\nu_{t, x}, \nu_{t, x}^{\infty}, \lambda\right)$ is $\left(\mathfrak{F}_{t}\right)$-adapted, we have $\mathcal{V} \in Y_{2}^{\text {loc }}\left(Q_{\infty}\right) \mathbb{P}$-a.s. and $\lambda=\lambda_{t} \otimes \mathscr{L}^{1}$ with $\lambda_{t} \in$ $L_{w^{*}}^{\infty}\left(0, T ; \mathscr{M}^{+}\left(\mathbb{T}^{3}\right)\right) \mathbb{P}$-a.s.;

(e) We have $\mathbf{u}(t, x)=\left\langle\nu_{t, x}, \boldsymbol{\xi}\right\rangle \mathbb{P}$-a.s. for a.e. $(t, x) \in Q_{\infty}$;

(f) $\Lambda=\mathbb{P} \circ(\mathbf{u}(0))^{-1}$ and $\mathcal{L}[\mathbf{u}(0), \mathcal{V}, \mathbf{u}, W]$ is a Radon measure on $(\mathcal{X}, \mathscr{B} \mathcal{X})$;

(g) For all $\varphi \in C_{\mathrm{div}}^{\infty}\left(\mathbb{T}^{3}\right)$ and all $t \geq 0$ there holds $\mathbb{P}$-a.s.

$$
\begin{aligned}
\int_{\mathbb{T}^{3}} \mathbf{u}(t) \cdot \boldsymbol{\varphi} \mathrm{d} x= & \int_{\mathbb{T}^{3}} \mathbf{u}(0) \cdot \boldsymbol{\varphi} \mathrm{d} x+\int_{0}^{t} \int_{\mathbb{T}^{3}}\left\langle\nu_{t, x}, \boldsymbol{\xi} \otimes \boldsymbol{\xi}\right\rangle: \nabla \boldsymbol{\varphi} \mathrm{d} x \mathrm{~d} s \\
& +\int_{(0, t) \times \mathbb{T}^{3}}\left\langle\nu_{t, x}^{\infty}, \boldsymbol{\xi} \otimes \boldsymbol{\xi}\right\rangle: \nabla \boldsymbol{\varphi} \mathrm{d} \lambda+\int_{0}^{t} \int_{\mathbb{T}^{3}} \boldsymbol{\varphi} \cdot \Phi \mathrm{d} x \mathrm{~d} W ;
\end{aligned}
$$

(h) The energy inequality holds in the sense that

$$
E_{t^{+}} \leq E_{s^{-}}+\frac{1}{2} \int_{s}^{t}\|\Phi\|_{L_{2}\left(\left(\mathfrak{U}, L^{2}\left(\mathbb{T}^{3}\right)\right)\right)}^{2} \mathrm{~d} \sigma+\int_{s}^{t} \int_{\mathbb{T}^{3}} \mathbf{u} \cdot \Phi \mathrm{d} x \mathrm{~d} W
$$

$\mathbb{P}$-a.s. for all $0 \leq s<t$, where $E_{t}=\frac{1}{2} \int_{\mathbb{T}^{3}}\left\langle\nu_{t, x},|\boldsymbol{\xi}|^{2}\right\rangle \mathrm{d} x+\frac{1}{2} \lambda_{t}\left(\mathbb{T}^{3}\right)$ for $t \geq 0$ with $\lambda=\lambda_{t} \otimes \mathcal{L}^{1}$ and $E_{0^{-}}=\frac{1}{2} \int_{\mathbb{T}^{3}}|\mathbf{u}(0)|^{2} \mathrm{~d} x$.

Remark 3.2. Some remark concerning the energy inequality (3.5) are in order. At first glance it is not clear why the left- and right-sided limits

$$
E_{t^{+}}=\lim _{\tau \searrow t} E_{\tau}, \quad E_{t^{-}}=\lim _{\tau \nearrow t} E_{\tau}
$$

exists in any time-point. Initially, we only show that

$$
E_{t} \leq E_{s}+\frac{1}{2} \int_{s}^{t}\|\Phi\|_{L_{2}\left(\left(\mathfrak{U}, L^{2}\left(\mathbb{T}^{3}\right)\right)\right)}^{2} \mathrm{~d} \sigma+\int_{s}^{t} \int_{\mathbb{T}^{3}} \mathbf{u} \cdot \Phi \mathrm{d} x \mathrm{~d} W
$$

$\mathbb{P}$-a.s. for a.a. $0<s<t$, see (3.21). This, however, implies that the mapping

$$
t \mapsto E_{t}-\int_{0}^{t}\|\Phi\|_{L_{2}\left(\left(\mathfrak{U}, L^{2}\left(\mathbb{T}^{3}\right)\right)\right)}^{2} \mathrm{~d} \sigma-\int_{0}^{t} \int_{\mathbb{T}^{3}} \mathbf{u} \cdot \Phi \mathrm{d} x \mathrm{~d} W
$$

is non-increasing. Since it is also pathwise bounded, left- and right-sided limits exist in all points. Furthermore, $\int_{0}^{*}\|\Phi\|_{L_{2}\left(\left(\mathfrak{U}, L^{2}\left(\mathbb{T}^{3}\right)\right)\right)}^{2} \mathrm{~d} t$ and $\int_{0}^{\cdot} \int_{\mathbb{T}^{3}} \mathbf{u} \cdot \Phi \mathrm{d} x \mathrm{~d} W$ are continuous such that left- and right-sided limits also exist for $E_{t}$. Finally, we obtain $E_{t^{+}} \leq E_{t^{-}}$, such that there could be energetic sinks but no positive jumps in the energy.

The main result of this section concerns the existence of a dissipative solution in the sense of Definition 3.1 .

Theorem 3.3. Assume that we have

$$
\int_{L_{\mathrm{div}}^{2}\left(\mathbb{T}^{3}\right)}\|\mathbf{w}\|_{L_{x}^{2}}^{p} \mathrm{~d} \Lambda(\mathbf{w})<\infty
$$

for some $p>2$. Then there is a dissipative martingale solution to (3.1) in the sense of Definition 3.1. 
As a by-product of our proof, in which we approximate (3.1) by a sequence of solutions to (2.15) with vanishing viscosity, we obtain the following result.

Corollary 3.4. Let $\Lambda$ be a given Borel probability measure on $L_{\mathrm{div}}^{2}\left(\mathbb{T}^{3}\right)$ such that

$$
\int_{L_{\mathrm{div}}^{2}\left(\mathbb{T}^{3}\right)}\|\mathbf{w}\|_{L_{x}^{2}}^{p} \mathrm{~d} \Lambda(\mathbf{w})<\infty
$$

for some $p>2$. If $\left(\left(\Omega^{\varepsilon}, \mathfrak{F}^{\varepsilon},\left(\mathfrak{F}^{\varepsilon}\right), \mathbb{P}^{\varepsilon}\right), \mathbf{u}^{\varepsilon}, W^{\varepsilon}\right)$ is a finite energy weak martingale solution to (2.15) in the sense of Definition 2.6 with the initial law $\Lambda$, then there is a subsequence such that

$$
\mathbf{u}^{\varepsilon} \rightarrow \mathbf{u} \quad \text { in law on } \quad C_{w, \text { loc }}\left([0, \infty) ; L_{\text {div }}^{2}\left(\mathbb{T}^{3}\right)\right)
$$

where $\mathbf{u}$ is a dissipative solution to (3.1) in the sense of Definition 3.1 with the initial law $\Lambda$.

The rest of this section is dedicated to the proof of Theorem 3.3 which we split in several parts.

\subsection{A Priori Estimates}

For any $\varepsilon>0$ Theorem 2.7 yields the existence of a martingale solution

$$
\left(\left(\Omega^{\varepsilon}, \mathfrak{F}^{\varepsilon},\left(\mathfrak{F}_{t}^{\varepsilon}\right), \mathbb{P}^{\varepsilon}\right), \mathbf{u}^{\varepsilon}, W^{\varepsilon}\right)
$$

to (2.15). Without loss of generality we can assume that the probability space does not depend on $\varepsilon$, that is the solution is given by

$$
\left(\left(\Omega, \mathfrak{F},\left(\mathfrak{F}_{t}^{\varepsilon}\right), \mathbb{P}\right), \mathbf{u}^{\varepsilon}, W^{\varepsilon}\right) .
$$

Indeed, since martingale solutions are constructed by the stochastic compactness method based on Skorokhod's theorem we may consider

$$
\left(\Omega^{\varepsilon}, \mathfrak{F}^{\varepsilon}, \mathbb{P}^{\varepsilon}\right)=\left([0,1], \overline{\mathscr{B}([0,1])},\left.\mathscr{L}^{1}\right|_{[0,1]}\right)
$$

as shown, for instance, in [26].

From (2.16) we obtain for any $T>0$ (choosing $\phi=\mathbb{I}_{(0, t)}$ and $s=0$, taking the supremum with respect to $t$, the power $p$ and applying expectations)

$$
\begin{aligned}
& \mathbb{E}\left[\sup _{0<t<T} \int_{\mathbb{T}^{3}}\left|\mathbf{u}^{\varepsilon}\right|^{2} \mathrm{~d} x+\varepsilon \int_{0}^{T} \int_{\mathbb{T}^{3}}\left|\nabla \mathbf{u}^{\varepsilon}\right|^{2} \mathrm{~d} x \mathrm{~d} t\right]^{p} \\
& \leq c(p) \int_{L_{\mathrm{div}}^{2}\left(\mathbb{T}^{3}\right)}\|\mathbf{w}\|_{L_{x}^{2}}^{2 p} \mathrm{~d} \Lambda(\mathbf{w})+c(p) \mathbb{E}\left[\sup _{0<t<T} \int_{\mathbb{T}^{3}} \int_{0}^{t} \mathbf{u}^{\varepsilon} \cdot \Phi \mathrm{d} W^{\varepsilon} \mathrm{d} x\right]^{p} \\
& \quad+c(p) \mathbb{E}\left[\int_{0}^{T}\|\Phi\|_{L_{2}\left(\mathfrak{U} ; L^{2}\left(\mathbb{T}^{3}\right)\right)}^{2} \mathrm{~d} t\right]^{p} .
\end{aligned}
$$

By Burkholder-Davis-Gundy inequality we obtain

$$
\begin{aligned}
E\left[\sup _{0<t<T} \int_{\mathbb{T}^{3}} \int_{0}^{t} \mathbf{u}^{\varepsilon} \cdot \Phi \mathrm{d} W^{\varepsilon} \mathrm{d} x\right]^{p} & \leq c(p) \mathbb{E}\left[\int_{0}^{T} \sum_{k \geq 1}\left(\int_{\mathbb{T}^{3}} \Phi \mathbf{e}_{k} \cdot \mathbf{u}^{\varepsilon} \mathrm{d} x\right)^{2}\right]^{\frac{p}{2}} \\
& \leq c(p) \mathbb{E}\left[\|\Phi\|_{L_{2}\left(\mathfrak{U} ; L^{2}\left(\mathbb{T}^{3}\right)\right)}^{2} \int_{0}^{T} \int_{\mathbb{T}^{3}}\left|\mathbf{u}^{\varepsilon}\right|^{2} \mathrm{~d} x \mathrm{~d} t\right]^{\frac{p}{2}} \\
& \leq c(p, \Phi, T) \int_{0}^{T} \mathbb{E}\left[\int_{\mathbb{T}^{3}}\left|\mathbf{u}^{\varepsilon}\right|^{2} \mathrm{~d} x\right]^{\frac{p}{2}} \mathrm{~d} t .
\end{aligned}
$$

By Gronwall's lemma we conclude

$$
\mathbb{E}\left[\sup _{0<t<T} \int_{\mathbb{T}^{3}}\left|\mathbf{u}^{\varepsilon}\right|^{2} \mathrm{~d} x+\varepsilon \int_{0}^{T} \int_{\mathbb{T}^{3}}\left|\nabla \mathbf{u}^{\varepsilon}\right|^{2} \mathrm{~d} x \mathrm{~d} t\right]^{p} \leq c(p, \Lambda, \Phi, T)
$$


uniformly in $\varepsilon$. We have to pass to the limit in the nonlinear convective term which requires some compactness arguments. We write the momentum equation as

$$
\begin{aligned}
\int_{\mathbb{T}^{3}} \mathbf{u}^{\varepsilon}(t) \cdot \boldsymbol{\varphi} \mathrm{d} x & =\int_{\mathbb{T}^{3}} \mathbf{u}^{\varepsilon}(0) \cdot \boldsymbol{\varphi} \mathrm{d} x+\int_{0}^{t} \int_{\mathbb{T}^{3}} \mathbf{H}^{\varepsilon}: \nabla \boldsymbol{\varphi} \mathrm{d} x \mathrm{~d} \sigma+\int_{0}^{t} \int_{\mathbb{T}^{3}} \boldsymbol{\varphi} \cdot \Phi \mathrm{d} x \mathrm{~d} W^{\varepsilon}, \\
\mathbf{H}^{\varepsilon} & :=-\varepsilon \nabla \mathbf{u}^{\varepsilon}+\mathbf{u}^{\varepsilon} \otimes \mathbf{u}^{\varepsilon}
\end{aligned}
$$

for all $\varphi \in C_{\text {div }}^{\infty}\left(\mathbb{T}^{3}\right)$. From the a priori estimates in 3.6 we obtain

$$
\mathbf{H}^{\varepsilon} \in L^{1}\left(\Omega ; L^{2}\left(0, T ; L^{1}\left(\mathbb{T}^{3}\right)\right) \hookrightarrow L^{1}\left(\Omega ; L^{2}\left(0, T ; W^{-2,2}\left(\mathbb{T}^{3}\right)\right)\right.\right.
$$

uniformly in $\varepsilon$. Let us consider the functional

$$
\mathscr{H}^{\varepsilon}(t, \varphi):=\int_{0}^{t} \int_{\mathbb{T}^{3}} \mathbf{H}^{\varepsilon}: \nabla \varphi \mathrm{d} x \mathrm{~d} \sigma, \quad \varphi \in C_{\mathrm{div}}^{\infty}\left(\mathbb{T}^{3}\right),
$$

which is the deterministic part of the equation. Then we deduce from (3.7) the estimate

$$
\mathbb{E}\left[\left\|\mathscr{H}^{\varepsilon}\right\|_{W^{1,2}\left(0, T ; W_{\text {div }}^{-3,2}\left(\mathbb{T}^{3}\right)\right)}\right] \leq c(T) .
$$

For the stochastic term we have

$$
\mathbb{E}\left[\left\|\int_{0}^{.} \Phi \mathrm{d} W^{\varepsilon}\right\|_{C^{\alpha}\left([0, T] ; L^{2}\left(\mathbb{T}^{3}\right)\right)}^{p}\right] \leq c \mathbb{E}\left[\int_{0}^{T}\|\Phi\|_{L_{2}\left(\mathfrak{U}, L^{2}\left(\mathbb{T}^{3}\right)\right)}^{p} \mathrm{~d} t\right]=c(p, \Phi, T)
$$

for all $\alpha \in(0,1 / 2-1 / p)$ and $p>2$. Combining the two previous estimates and using the embeddings $W_{t}^{1,2} \hookrightarrow C_{t}^{1 / 2}$ and $L_{x}^{2} \hookrightarrow W_{x}^{-3,2}$ shows

$$
\mathbb{E}\left[\left\|\mathbf{u}^{\varepsilon}\right\|_{C^{\alpha}\left([0, T] ; W_{\mathrm{div}}^{-3,2}\left(\mathbb{T}^{3}\right)\right)}\right] \leq c(\alpha, T)
$$

for all $\alpha<\frac{1}{2}$.

\subsection{Compactness}

We aim at proving tightness of the sequence of approximate solutions using the compact embeddings

$$
\begin{aligned}
C^{\alpha}\left([0, T] ; W_{\text {div }}^{-3,2}\left(\mathbb{T}^{3}\right)\right) & \hookrightarrow \hookrightarrow C\left([0, T] ; W_{\text {div }}^{-4,2}\left(\mathbb{T}^{3}\right)\right), \\
C^{\alpha}\left([0, T] ; W_{\text {div }}^{-3,2}\left(\mathbb{T}^{3}\right)\right) \cap L^{\infty}\left(0, T ; L_{\text {div }}^{2}\left(\mathbb{T}^{3}\right)\right) & \hookrightarrow C_{w}\left([0, T] ; L_{\text {div }}^{2}\left(\mathbb{T}^{3}\right)\right) .
\end{aligned}
$$

For $T>0$ we consider the path space

$$
\begin{aligned}
\mathcal{X}_{T}:= & L_{\mathrm{div}}^{2}\left(\mathbb{T}^{3}\right) \times C\left([0, T] ; W_{\mathrm{div}}^{-4,2}\left(\mathbb{T}^{3}\right)\right) \cap C_{w}\left([0, T] ; L_{\mathrm{div}}^{2}\left(\mathbb{T}^{3}\right)\right) \times Y_{2}\left(Q_{T}\right) \\
& \times C\left([0, T], \mathfrak{U}_{0}\right) .
\end{aligned}
$$

Clearly, tightness of $\mathcal{L}\left[\mathbf{u}_{0}, \mathbf{r}_{T} \mathbf{u}^{\varepsilon_{m}}, \mathbf{r}_{T} \mathcal{V}^{\varepsilon_{m}}, \mathbf{r}_{T} W^{\varepsilon}\right]$ on $\mathcal{X}_{T}$ for any $T>0$ implies tightness of $\mathcal{L}\left[\mathbf{u}_{0}, \mathbf{u}^{\varepsilon_{m}}, \mathcal{V}^{\varepsilon_{m}}, W^{\varepsilon}\right]$ on $\mathcal{X}$. Here $\mathbf{r}_{T}$ is the restriction operator which restricts measurable functions (or space-time distributions) defined on $(0, \infty)$ to $(0, T)$. It acts on various path spaces. We fix $T>0$ and consider the ball $\mathcal{B}_{R}$ in the space

$$
C^{\alpha}\left([0, T] ; W_{\operatorname{div}}^{-3,2}\left(\mathbb{T}^{3}\right)\right) \cap L^{\infty}\left(0, T ; L_{\operatorname{div}}^{2}\left(\mathbb{T}^{3}\right)\right) .
$$

We obtain for its complement by (3.6) and (3.8)

$$
\mathcal{L}\left[\mathbf{r}_{T} \mathbf{u}^{\varepsilon}\right]\left(\mathcal{B}_{R}^{C}\right)=\mathbb{P}\left(\left\|\mathbf{r}_{T} \mathbf{u}^{\varepsilon}\right\|_{C_{t}^{\alpha} W_{x}^{-3,2}}+\left\|\mathbf{r}_{T} \mathbf{u}^{\varepsilon}\right\|_{L_{t}^{\infty} L_{x}^{2}} \geq R\right) \leq \frac{c}{R} .
$$

So, for any fixed $\eta>0$, we find $R(\eta)$ with

$$
\mathcal{L}\left[\mathbf{r}_{T} \mathbf{u}^{\varepsilon}\right]\left(\mathcal{B}_{R(\eta)}\right) \geq 1-\eta,
$$


i.e. $\mathcal{L}\left[\mathbf{r}_{T} \mathbf{u}^{\varepsilon}\right]$ is tight. Now we set $\mathcal{V}^{\varepsilon}=\left(\delta_{\mathbf{u}^{\varepsilon}}, 0,0\right) \in Y_{2}^{\text {loc }}\left(Q_{\infty}\right)$ as the generalised Young measure associated to $\mathbf{u}^{\varepsilon}$. Similarly to the above we have

$$
\mathcal{L}\left[\mathbf{r}_{T} \mathbf{u}^{\varepsilon}\right]\left(\mathcal{B}_{R(\eta)}\right) \geq 1-\eta
$$

for some $R=R(\eta)$, where $\mathcal{B}_{R(\eta)}$ is now the ball in $L^{\infty}\left(0, T ; L^{2}\left(\mathbb{T}^{3}\right)\right)$. Recalling (2.3) we conclude tightness of $\mathcal{L}\left[\mathbf{r}_{T} \mathcal{V}^{\varepsilon}\right]$.

Since also the laws $\mathcal{L}\left[\mathbf{r}_{T} W^{\varepsilon}\right]$ and $\mathcal{L}\left[\mathbf{u}_{0}\right]$ are tight, as being Radon measures on the Polish spaces $C\left([0, T], \mathfrak{U}_{0}\right)$ and $L_{\text {div }}^{2}\left(\mathbb{T}^{3}\right)$, we can conclude that $\mathcal{L}\left[\mathbf{u}_{0}, \mathbf{r}_{T} \mathbf{u}^{\varepsilon}, \mathbf{r}_{T} \mathcal{V}^{\varepsilon}, \mathbf{r}_{T} W^{\varepsilon}\right]$ is tight on $\mathcal{X}_{T}$. Since $T$ was arbitrary we conclude that $\mathcal{L}\left[\mathbf{u}_{0}, \mathbf{u}^{\varepsilon}, \mathcal{V}^{\varepsilon}, W^{\varepsilon}\right]$ is tight on $\mathcal{X}$. Now we use Jakubowski's version of the Skorokhod representation theorem, see [26], to infer the following result ${ }^{1}$ (we refer to [34, Theorem A.1] for a statement which combines Prokhorov's and Skorokhod's theorem for quasi-Polish spaces). Let us remark that $\mathcal{L}\left[\mathbf{u}_{0}, \mathbf{u}^{\varepsilon_{m}}, \mathcal{V}^{\varepsilon_{m}}, W^{\varepsilon}\right]$ is a sequence of tight measures on $(\mathcal{X}, \mathscr{B} \mathcal{X})$. Consequently, its weak* limit is tight as well and hence Radon.

Proposition 3.5. There exists a nullsequence $\left(\varepsilon_{m}\right)_{m \in \mathbb{N}}$, a complete probability space $(\tilde{\Omega}, \tilde{\mathfrak{F}}, \tilde{\mathbb{P}})$ with $(\mathcal{X}, \mathscr{B} \mathcal{X})$ valued random variables $\left(\tilde{\mathbf{u}}_{0}^{\varepsilon_{m}}, \tilde{\mathbf{u}}^{\varepsilon_{m}}, \tilde{\mathcal{V}}^{\varepsilon_{m}}, \tilde{W}^{\varepsilon_{m}}\right), m \in \mathbb{N}$, and $\left(\tilde{\mathbf{u}}_{0}, \tilde{\mathbf{u}}, \tilde{\mathcal{V}}, \tilde{W}\right)$ such that

(a) For all $m \in N$ the law of $\left(\tilde{\mathbf{u}}_{0}^{\varepsilon_{m}}, \tilde{\mathbf{u}}^{\varepsilon_{m}}, \tilde{\mathcal{V}}^{\varepsilon_{m}}, \tilde{W}^{\varepsilon_{m}}\right)$ on $\mathcal{X}$ is given by $\mathcal{L}\left[\mathbf{u}_{0}, \mathbf{u}^{\varepsilon_{m}}, \mathcal{V}^{\varepsilon_{m}}, W^{\varepsilon_{m}}\right]$;

(b) The law of $\left(\tilde{\mathbf{u}}_{0}, \tilde{\mathbf{u}}, \tilde{\mathcal{V}}, \tilde{W}\right)$ is a Radon measure on $(\mathcal{X}, \mathscr{B} \mathcal{X})$;

(c) $\left(\tilde{\mathbf{u}}_{0}^{\varepsilon_{m}}, \tilde{\mathbf{u}}^{\varepsilon_{m}}, \tilde{\mathcal{V}}^{\varepsilon_{m}}, \tilde{W}^{\varepsilon_{m}}\right)$ converges $\tilde{\mathbb{P}}$-almost surely to $\left(\tilde{\mathbf{u}}_{0}, \tilde{\mathbf{u}}, \tilde{\mathcal{V}}, \tilde{W}\right)$ in the topology of $\mathcal{X}$, i.e.

$$
\begin{aligned}
& \tilde{\mathbf{u}}_{0}^{\varepsilon_{m}} \rightarrow \tilde{\mathbf{u}}_{0} \quad \text { in } \quad L^{2}\left(\mathbb{T}^{3}\right) \tilde{\mathbb{P}} \text {-a.s. }, \\
& \tilde{\mathbf{u}}^{\varepsilon_{m}} \rightarrow \tilde{\mathbf{u}} \quad \text { in } \quad C_{\text {loc }}\left([0, \infty) ; W_{\operatorname{div}}^{-4,2}\left(\mathbb{T}^{3}\right)\right) \tilde{\mathbb{P}} \text {-a.s. }, \\
& \tilde{\mathbf{u}}^{\varepsilon_{m}} \rightarrow \tilde{\mathbf{u}} \quad \text { in } \quad C_{w, l o c}\left([0, \infty) ; L_{\operatorname{div}}^{2}\left(\mathbb{T}^{3}\right)\right) \tilde{\mathbb{P}} \text {-a.s. }, \\
& \tilde{\mathcal{V}}^{\varepsilon_{m}} \rightarrow^{*} \tilde{\mathcal{V}} \quad \text { in } \quad Y_{2}^{\text {loc }}\left(Q_{\infty}\right) \tilde{\mathbb{P}} \text {-a.s. }, \\
& \tilde{W}^{\varepsilon_{m}} \rightarrow \tilde{W} \quad \text { in } \quad C_{\mathrm{loc}}\left([0, \infty) ; \mathfrak{U}_{0}\right) \tilde{\mathbb{P}} \text {-a.s. }
\end{aligned}
$$

It is now easy to show that we have $\tilde{\mathbb{P}}$-a.s.

$$
\tilde{\mathbf{u}}^{\varepsilon_{m}}(t, x)=\left\langle\tilde{\nu}_{t, x}^{\varepsilon_{m}}, \boldsymbol{\xi}\right\rangle, \quad \tilde{\mathbf{u}}(t, x)=\left\langle\tilde{\nu}_{t, x}, \boldsymbol{\xi}\right\rangle \quad \text { for a.a. } \quad(t, x) \in Q_{\infty},
$$

where $\tilde{\mathcal{V}}^{\varepsilon_{m}}=\left(\tilde{\nu}_{t, x}^{\varepsilon_{m}}, \tilde{\nu}_{t, x}^{\infty, \varepsilon_{m}}, \tilde{\lambda}^{\varepsilon_{m}}\right)$ and $\tilde{\mathcal{V}}=\left(\tilde{\nu}_{t, x}, \tilde{\nu}_{t, x}^{\infty}, \tilde{\lambda}\right)$. Indeed, for $T>0$ and $\boldsymbol{\psi} \in C_{c}^{\infty}\left(Q_{T}\right)$ we consider the mapping

$$
(\mathbf{w}, \mathcal{V}) \mapsto \int_{Q_{T}}\left(\mathbf{w}-\left\langle\nu_{t, x}, \boldsymbol{\xi}\right\rangle\right) \cdot \boldsymbol{\psi} \mathrm{d} x \mathrm{~d} t
$$

which is continuous on the paths space. We obtain from Proposition 3.5

$$
\int_{Q_{T}}\left(\tilde{\mathbf{u}}^{\varepsilon_{m}}-\left\langle\tilde{\nu}_{t, x}^{\varepsilon_{m}}, \boldsymbol{\xi}\right\rangle\right) \cdot \boldsymbol{\psi} \mathrm{d} x \mathrm{~d} t \sim^{d} \int_{Q_{T}}\left(\mathbf{u}^{\varepsilon_{m}}-\left\langle\nu_{t, x}^{\varepsilon_{m}}, \boldsymbol{\xi}\right\rangle\right) \cdot \boldsymbol{\psi} \mathrm{d} x \mathrm{~d} t=0
$$

which implies the first claim from (3.11) by arbitrariness of $\boldsymbol{\psi}$ and $T$. Using again Proposition 3.5 we can pass to the limit $m \rightarrow \infty$ and the second assertion follows. Similarly, for any $T>0$ we can consider for $f \in \mathcal{G}_{2}\left(Q_{T}\right)$ and $\varphi \in C\left(\bar{Q}_{T}\right)$ arbitrary the mappings

$$
(\mathbf{w}, \mathcal{V}) \mapsto \int_{Q_{T}} \varphi\left\langle\nu_{t, x}-\delta_{\mathbf{w}(t, x)}, f(\boldsymbol{\xi})\right\rangle \mathrm{d} x \mathrm{~d} t+\int_{Q_{T}} \varphi\left\langle\nu_{t, x}^{\infty}, f^{\infty}(\boldsymbol{\xi})\right\rangle \mathrm{d} \lambda
$$

to show that ${ }^{2}$

$$
\tilde{\mathcal{V}}^{\varepsilon_{m}}=\left(\tilde{\nu}_{t, x}^{\varepsilon_{m}}, \tilde{\nu}_{t, x}^{\infty, \varepsilon_{m}}, \tilde{\lambda}^{\varepsilon_{m}}\right)=\left(\delta_{\tilde{\mathbf{u}}^{\varepsilon_{m}}(t, x)}, 0,0\right) \text { for a.a. } \quad(t, x) \in Q_{\infty}
$$

\footnotetext{
${ }^{1}$ To be precise, one first has to replace the family of random variables indexed by $\varepsilon$ by a countable sub-family.

${ }^{2}$ Notice that we have $\left(\delta_{\tilde{\mathbf{u}}^{\varepsilon} m(t, x)}, 0,0\right)=\left(\delta_{\tilde{\mathbf{u}}^{\varepsilon} m(t, x)}, \tilde{\nu}_{t, x}^{\varepsilon_{m}}, 0\right)=\left(\delta_{\tilde{\mathbf{u}}^{\varepsilon} m(t, x)}, 0, \tilde{\lambda}\right)$ in the sense of generalised Young measures.
} 
Now we introduce the filtration on the new probability space, which ensures the correct measurabilities

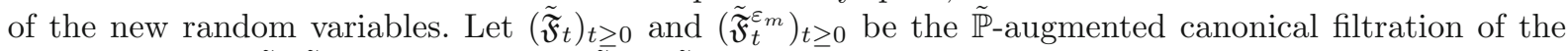
variables $\left(\tilde{\mathbf{u}}_{0}, \tilde{\mathbf{u}}, \tilde{\mathcal{V}}, \tilde{W}\right)$ and $\left(\tilde{\mathbf{u}}_{0}^{\varepsilon_{m}}, \tilde{\mathbf{u}}^{\varepsilon_{m}}, \tilde{\mathcal{V}}^{\varepsilon_{m}}, \tilde{W}^{\varepsilon_{m}}\right)$, respectively, that is

$$
\begin{aligned}
\tilde{\mathfrak{F}}_{t} & =\sigma\left(\sigma\left(\tilde{\mathbf{u}}_{0}, \mathbf{r}_{t} \tilde{\mathbf{u}}, \mathbf{r}_{t} \tilde{W}\right) \cup \sigma_{t}[\tilde{\mathcal{V}}] \cup\{\mathcal{N} \in \tilde{\mathfrak{F}} ; \tilde{\mathbb{P}}(\mathcal{N})=0\}\right), \quad t \geq 0, \\
\tilde{\mathfrak{F}}_{t}^{\varepsilon_{m}} & =\sigma\left(\sigma\left(\tilde{\mathbf{u}}_{0}^{\varepsilon_{m}}, \tilde{\mathbf{u}}^{\varepsilon_{m}}, \mathbf{r}_{t} \tilde{W}^{\varepsilon_{m}}\right) \cup \sigma_{t}\left[\tilde{\mathcal{V}}^{\varepsilon_{m}}\right] \cup\{\mathcal{N} \in \tilde{\mathfrak{F}} ; \tilde{\mathbb{P}}(\mathcal{N})=0\}\right), \quad t \geq 0 .
\end{aligned}
$$

Here $\sigma_{t}$ denotes the history of a random distribution as defined in (2.6), where generalised Young measures are identified as random distribution in the sense of (2.4). The definitions above guarantee that the processes are adapted and we can define stochastic integrals.

\subsection{Concerning the New Probability Space}

Now are going to show that the approximated equations also hold on the new probability space. We use the elementary method from [9] which has already been generalized to different settings (see, for instance, $[6,25])$. The key idea is to identify the quadratic variation of the corresponding martingale as well as its cross variation with the limit Wiener process obtained through compactness. First we notice that $\tilde{W}$ has the same law as $W$. As a consequence, there exists a collection of mutually independent real-valued $\left(\tilde{\mathfrak{F}}_{t}^{\varepsilon_{m}}\right)_{t \geq 0}$-Wiener processes $\left(\tilde{\beta}_{k}^{\varepsilon_{m}}\right)$ such that $\tilde{W}^{N}=\sum_{k} \tilde{\beta}_{k}^{\varepsilon_{m}} e_{k}$. In particular, there exists a collection of mutually independent real-valued $\left(\tilde{\mathfrak{F}}_{t}\right)_{t \geq 0}$-Wiener processes $\left(\tilde{\beta}_{k}\right)$ such that $\tilde{W}=\sum_{k} \tilde{\beta}_{k} e_{k}$. Let us now define for all $t \in[0, T]$ and $\varphi \in C_{\operatorname{div}}^{\infty}\left(\mathbb{T}^{3}\right)$ the functionals

$$
\begin{aligned}
\mathfrak{M}^{\varepsilon_{m}}\left(\mathbf{u}_{0}, \mathbf{u}, \mathcal{V}\right)_{t}= & \int_{\mathbb{T}^{3}}\left(\mathbf{u}(t)-\mathbf{u}_{0}\right) \cdot \boldsymbol{\varphi} \mathrm{d} x-\varepsilon_{m} \int_{0}^{t} \int_{\mathbb{T}^{3}} \mathbf{u} \cdot \Delta \boldsymbol{\varphi} \mathrm{d} x \mathrm{~d} s \\
& -\int_{0}^{t} \int_{\mathbb{T}^{3}}\left\langle\nu_{t, x}, \boldsymbol{\xi} \otimes \boldsymbol{\xi}\right\rangle: \nabla \boldsymbol{\varphi} \mathrm{d} x \mathrm{~d} s-\int_{(0, t) \times \mathbb{T}^{3}}\left\langle\nu_{t, x}^{\infty}, \boldsymbol{\xi} \otimes \boldsymbol{\xi}\right\rangle: \nabla \boldsymbol{\varphi} \mathrm{d} \lambda \\
\mathfrak{N}_{t}= & \sum_{k=1}^{\infty} \int_{0}^{t}\left(\int_{\mathbb{T}^{3}} \Phi \mathbf{e}_{k} \cdot \boldsymbol{\varphi} \mathrm{d} x\right)^{2} \mathrm{~d} \sigma, \quad \mathfrak{N}_{t}^{k}=\int_{0}^{t} \int_{\mathbb{T}^{3}} \Phi \mathbf{e}_{k} \cdot \boldsymbol{\varphi} \mathrm{d} x \mathrm{~d} \sigma .
\end{aligned}
$$

By $\mathfrak{M}\left(\mathbf{u}^{\varepsilon_{m}}(0), \mathbf{u}^{\varepsilon_{m}}, \mathcal{V}\right)_{s, t}$ we denote the increment $\mathfrak{M}\left(\mathbf{u}^{\varepsilon_{m}}(0), \mathbf{u}^{\varepsilon_{m}}, \mathcal{V}\right)_{t}-\mathfrak{M}\left(\mathbf{u}^{\varepsilon_{m}}(0), \mathbf{u}^{\varepsilon_{m}}, \mathcal{V}\right)_{s}$ and similarly for $\mathfrak{N}_{s, t}$ and $\mathfrak{N}_{s, t}^{k}$. Note that the proof will be complete once we show that the process $\mathfrak{M}\left(\tilde{\mathbf{u}}_{0}^{\varepsilon_{m}}, \tilde{\mathbf{u}}^{\varepsilon_{m}}, \tilde{\mathcal{V}}^{\varepsilon_{m}}\right)$ is an $\left(\tilde{\mathfrak{F}}_{t}^{\varepsilon_{m}}\right)_{t \geq 0}$-martingale and its quadratic and cross variations satisfy, respectively,

$$
\left\langle\left\langle\mathfrak{M}\left(\tilde{\mathbf{u}}_{0}^{\varepsilon_{m}}, \tilde{\mathbf{u}}^{\varepsilon_{m}}, \tilde{\mathcal{V}}^{\varepsilon_{m}}\right)\right\rangle\right\rangle=\mathfrak{N}, \quad\left\langle\left\langle\mathfrak{M}\left(\tilde{\mathbf{u}}_{0}^{\varepsilon_{m}}, \tilde{\mathbf{u}}^{\varepsilon_{m}}, \tilde{\mathcal{V}}^{\varepsilon_{m}}\right), \tilde{\beta}_{k}\right\rangle\right\rangle=\mathfrak{N}^{k}
$$

Indeed, in that case we have

$$
\left\langle\left\langle\mathfrak{M}\left(\tilde{\mathbf{u}}_{0}^{\varepsilon_{m}}, \tilde{\mathbf{u}}^{\varepsilon_{m}}, \tilde{\mathcal{V}}^{\varepsilon_{m}}\right)-\int_{0}^{\cdot} \int_{\mathbb{T}^{3}} \varphi \cdot \Phi \mathrm{d} x \mathrm{~d} \tilde{W}^{\varepsilon_{m}}\right\rangle\right\rangle=0
$$

which implies the desired equation on the new probability space. Let us verify (3.13). To this end, we claim that with the above uniform estimates in hand, the mapping

$$
\left(\mathbf{u}_{0}, \mathbf{u}, \mathcal{V}\right) \mapsto \mathfrak{M}\left(\mathbf{u}_{0}, \mathbf{u}, \mathcal{V}\right)_{t}
$$

is well-defined and continuous on the path space. Hence we have

$$
\mathfrak{M}^{\varepsilon_{m}}\left(\mathbf{u}^{\varepsilon_{m}}(0), \mathbf{u}^{\varepsilon_{m}}, \mathcal{V}^{\varepsilon_{m}}\right) \sim^{d} \mathfrak{M}^{\varepsilon}\left(\tilde{\mathbf{u}}_{0}^{\varepsilon_{m}}, \tilde{\mathbf{u}}^{\varepsilon_{m}}, \tilde{\mathcal{V}}^{\varepsilon_{m}}\right) .
$$

Let us now fix times $s, t \in[0, T]$ such that $s<t$ and let

$$
h:\left.\mathcal{X}\right|_{[0, s]} \rightarrow[0,1]
$$


be a continuous function. Since

$$
\mathfrak{M}\left(\mathbf{u}^{\varepsilon_{m}}(0), \mathbf{u}^{\varepsilon_{m}}, \mathcal{V}^{\varepsilon_{m}}\right)_{t}=\int_{0}^{t} \int_{\mathbb{T}^{3}} \boldsymbol{\varphi} \cdot \Phi \mathrm{d} x \mathrm{~d} W^{\varepsilon_{m}}=\sum_{k=1}^{\infty} \int_{0}^{t} \int_{\mathbb{T}^{3}} \Phi \mathbf{e}_{k} \cdot \boldsymbol{\varphi} \mathrm{d} x \mathrm{~d} \beta_{k}^{\varepsilon_{m}}
$$

is a square integrable $\left(\mathfrak{F}_{t}\right)_{t \geq 0}$-martingale, we infer that

$$
\left[\mathfrak{M}^{\varepsilon_{m}}\left(\mathbf{u}^{\varepsilon_{m}}(0), \mathbf{u}^{\varepsilon_{m}}, \mathcal{V}^{\varepsilon_{m}}\right)\right]^{2}-\mathfrak{N}, \quad \mathfrak{M}^{\varepsilon_{m}}\left(\mathbf{u}^{\varepsilon_{m}}(0), \mathbf{u}^{\varepsilon_{m}}, \mathcal{V}^{\varepsilon_{m}}\right) \beta_{k}^{\varepsilon_{m}}-\mathfrak{N}^{k}
$$

are $\left(\mathfrak{F}_{t}\right)_{t>0}$-martingales. Let $\mathbf{r}_{s}$ be the restriction of a function to the interval $[0, s]$. Then it follows from the equality of laws in Proposition 3.5 that

$$
\begin{aligned}
& \tilde{\mathbb{E}}\left.h\left(\tilde{\mathbf{u}}_{0}^{\varepsilon_{m}}, \mathbf{r}_{s} \tilde{\mathbf{u}}^{\varepsilon_{m}}, \mathbf{r}_{s} \tilde{\mathcal{V}}^{\varepsilon_{m}}, \mathbf{r}_{s} \tilde{W}^{\varepsilon_{m}}\right) \mathfrak{M}^{\varepsilon_{m}}\left(\tilde{\mathbf{u}}_{0}^{\varepsilon_{m}}, \tilde{\mathbf{u}}^{\varepsilon_{m}}, \mathbf{r}_{s} \tilde{\mathcal{V}}^{\varepsilon_{m}}\right)_{s, t}\right] \\
&=\mathbb{E}\left[h\left(\mathbf{u}^{\varepsilon_{m}}(0), \mathbf{r}_{s} \mathbf{u}^{\varepsilon_{m}}, \mathbf{r}_{s} \mathcal{V}^{\varepsilon_{m}}, \mathbf{r}_{s} W^{\varepsilon_{m}}\right) \mathfrak{M}^{\varepsilon_{m}}\left(\mathbf{u}^{\varepsilon_{m}}(0), \mathbf{u}^{\varepsilon_{m}}, \mathcal{V}^{\varepsilon_{m}}\right)_{s, t}\right]=0, \\
& \tilde{\mathbb{E}}\left[h\left(\tilde{\mathbf{u}}_{0}^{\varepsilon_{m}}, \mathbf{r}_{s} \tilde{\mathbf{u}}^{\varepsilon_{m}}, \mathbf{r}_{s} \tilde{\mathcal{V}}^{\varepsilon_{m}}, \mathbf{r}_{s} \tilde{W}^{\varepsilon_{m}}\right)\left(\left[\mathfrak{M}^{\varepsilon_{m}}\left(\tilde{\mathbf{u}}_{0}^{\varepsilon_{m}}, \tilde{\mathbf{u}}^{\varepsilon_{m}}, \tilde{\mathcal{V}}^{\varepsilon_{m}}\right)^{2}\right]_{s, t}-\mathfrak{N}_{s, t}\right)\right] \\
& \quad=\mathbb{E}\left[h\left(\mathbf{u}^{\varepsilon_{m}}(0), \mathbf{r}_{s} \mathbf{u}^{\varepsilon_{m}}, \mathcal{V}^{\varepsilon_{m}}, \mathbf{r}_{s} W^{\varepsilon_{m}}\right)\left(\left[\mathfrak{M}^{\varepsilon_{m}}\left(\mathbf{u}^{\varepsilon_{m}}(0), \mathbf{u}^{\varepsilon_{m}}, \mathcal{V}^{\varepsilon_{m}}\right)^{2}\right]_{s, t}-\mathfrak{N}_{s, t}\right)\right]=0, \\
& \tilde{\mathbb{E}}\left[h\left(\tilde{\mathbf{u}}_{0}^{\varepsilon_{m}}, \mathbf{r}_{s} \tilde{\mathbf{u}}^{\varepsilon_{m}}, \mathbf{r}_{s} \tilde{\mathcal{V}}^{\varepsilon_{m}}, \mathbf{r}_{s} \tilde{W}^{\varepsilon_{m}}\right)\left(\left[\mathfrak{M}^{\varepsilon_{n}}\left(\tilde{\mathbf{u}}_{0}^{\varepsilon_{m}}, \tilde{\mathbf{u}}^{\varepsilon_{m}}, \tilde{\mathcal{V}}^{\varepsilon_{n}}\right) \tilde{\beta}_{k}^{\varepsilon_{m}}\right]_{s, t}-\mathfrak{N}_{s, t}^{k}\right)\right] \\
& \quad=\mathbb{E}\left[h\left(\mathbf{u}^{\varepsilon_{m}}(0), \mathbf{r}_{s} \mathbf{u}^{\varepsilon_{m}}, \mathbf{r}_{s} \mathcal{V}^{\varepsilon_{m}}, \mathbf{r}_{s} W^{\varepsilon_{m}}\right)\left(\left[\mathfrak{M}^{\varepsilon_{m}}\left(\mathbf{u}^{\varepsilon_{m}}(0), \mathbf{u}^{\varepsilon_{m}}, \mathcal{V}^{\varepsilon_{m}}\right) \beta_{k}^{\varepsilon_{m}}\right]_{s, t}-\mathfrak{N}_{s, t}^{k}\right)\right]=0 .
\end{aligned}
$$

So we have shown (3.13) and hence (3.14). On account of the convergences from Proposition 3.5 and the higher moments from (3.6) we can pass to the limit in (3.15)-(3.17) and obtain the momentum equation in the sense of (3.4).

Let us finally consider the energy inequality in the sense of (3.5), for which we introduce the abbreviations

$$
\mathscr{M}_{t}^{\varepsilon_{m}}=\int_{0}^{t} \int_{\mathbb{T}^{3}} \mathbf{u}^{\varepsilon_{m}} \cdot \Phi \mathrm{d} x \mathrm{~d} W^{\varepsilon_{m}}, \quad \tilde{\mathscr{M}}_{t}^{\varepsilon_{m}}=\int_{0}^{t} \int_{\mathbb{T}^{3}} \tilde{\mathbf{u}}^{\varepsilon_{m}} \cdot \Phi \mathrm{d} x \mathrm{~d} \tilde{W}^{\varepsilon_{m}},
$$

for the stochastic integrals. For the Navier-Stokes equations (on the original probability space) with $E_{t}^{\varepsilon_{m}}=\frac{1}{2} \int_{\mathbb{T}^{3}}\left|\mathbf{u}^{\varepsilon_{m}}\right|^{2} \mathrm{~d} x$ we have

$$
E_{t}^{\varepsilon_{m}} \leq E_{s}^{\varepsilon_{m}}+\frac{1}{2} \int_{s}^{t}\|\Phi\|_{L_{2}\left(\left(\mathfrak{U}, L^{2}\left(\mathbb{T}^{3}\right)\right)\right)}^{2} \mathrm{~d} \sigma+\mathscr{M}_{t}^{\varepsilon_{m}}-\mathscr{M}_{s}^{\varepsilon_{m}}
$$

for a.a. $s$ (including $s=0$ ) and all $t \geq s$, cf. (2.16). For a fixed $s$ this is equivalent to

$$
\begin{aligned}
& -\int_{s}^{\infty} \partial_{t} \phi E_{t}^{\varepsilon_{m}} \mathrm{~d} t-\phi(s) E_{s}^{\varepsilon_{m}} \\
& \quad \leq \frac{1}{2} \int_{s}^{\infty} \phi\|\Phi\|_{L_{2}\left(\left(\mathfrak{U}, L^{2}\left(\mathbb{T}^{3}\right)\right)\right)}^{2} \mathrm{~d} t+\int_{s}^{\infty} \phi \int_{\mathbb{T}^{3}} \mathbf{u}^{\varepsilon_{m}} \cdot \Phi \mathrm{d} x \mathrm{~d} W^{\varepsilon_{m}}
\end{aligned}
$$

$\mathbb{P}$-a.s. for all $\varphi \in C_{c}^{\infty}([s, \infty) ;[0, \infty))$. Due to Propositions 2.5 and 3.5 this continues to hold on the new probability space and we obtain

$$
\tilde{E}_{t}^{\varepsilon_{m}} \leq \tilde{E}_{s}^{\varepsilon_{m}}+\frac{1}{2} \int_{s}^{t}\|\Phi\|_{L_{2}\left(\left(\mathfrak{U}, L^{2}\left(\mathbb{T}^{3}\right)\right)\right)}^{2} \mathrm{~d} \sigma+\tilde{\mathscr{M}}_{t}^{\varepsilon_{m}}-\tilde{\mathscr{M}}_{s}^{\varepsilon_{m}}
$$

$\tilde{\mathbb{P}}$-a.s. for a.a. $s$ (including $s=0$ ) and all $t \geq s$. Averaging in $t$ and $s$ yields

$$
\begin{aligned}
f_{t-\varrho}^{t} \tilde{E}_{r}^{\varepsilon_{m}} \mathrm{~d} r \leq & f_{s-\varrho}^{s} \tilde{E}_{\tau}^{\varepsilon_{m}} \mathrm{~d} \tau+\frac{1}{2} f_{s-\varrho}^{s} f_{t-\varrho}^{t} \int_{\tau}^{r}\|\Phi\|_{L_{2}\left(\left(\mathfrak{U}, L^{2}\left(\mathbb{T}^{3}\right)\right)\right)}^{2} \mathrm{~d} \sigma \mathrm{d} r \mathrm{~d} \tau \\
& +f_{s-\varrho}^{s} f_{t-\varrho}^{t}\left(\tilde{\mathscr{M}}_{r}^{\varepsilon_{m}}-\tilde{\mathscr{M}}_{\tau}^{\varepsilon_{m}}\right) \mathrm{d} r \mathrm{~d} \tau
\end{aligned}
$$


provided $s>0$ and $\varrho<\min \{s, t-s\}$ (the easier case $s=0$ will be treated at the end). We aim to pass to the limit first in $m$ and then in $\varrho$. The terms in (3.18) involving the energy are continuous on the path space due to the additional time integrals. Hence they converge $\widetilde{\mathbb{P}}$-a.s. as $m \rightarrow \infty$ to the expected limits by Proposition 3.5. In order to prove that as $m \rightarrow \infty$ we have

$$
\tilde{\mathscr{M}}^{\varepsilon_{m}} \rightarrow \tilde{\mathscr{M}}:=\int_{0}^{t} \int_{\mathbb{T}^{3}} \tilde{\mathbf{u}} \cdot \Phi \mathrm{d} x \mathrm{~d} \tilde{W} \quad \text { in } \quad L_{\mathrm{loc}}^{2}([0, \infty))
$$

in probability we aim to apply [15, Lemma 2.1]. Hence we need to know in addition to $(3.10)_{5}$ that

$$
\int_{\mathbb{T}^{3}} \tilde{\mathbf{u}}^{\varepsilon_{m}} \cdot \Phi \mathrm{d} x \rightarrow \int_{\mathbb{T}^{3}} \tilde{\mathbf{u}} \cdot \Phi \mathrm{d} x \quad \text { in } \quad L_{\mathrm{loc}}^{2}\left([0, \infty) ; L_{2}(\mathfrak{U} ; \mathbb{R})\right)
$$

in probability. By $(3.10)_{3}$ we have $\tilde{\mathbb{P}}$-a.s.

$$
\int_{\mathbb{T}^{3}} \tilde{\mathbf{u}}^{\varepsilon_{m}}(t) \cdot \Phi \mathrm{d} x \rightarrow \int_{\mathbb{T}^{3}} \tilde{\mathbf{u}}(t) \cdot \Phi \mathrm{d} x \quad \text { in } \quad L_{2}(\mathfrak{U} ; \mathbb{R})
$$

for all $t \geq 0$. Hence we also obtain convergence in $L^{2}\left(\tilde{\Omega} ; L_{2}(\mathfrak{U} ; \mathbb{R})\right)$ using the higher moments from (3.6). Finally, we can use again (3.6) to obtain (3.20) (in fact, we even have $L^{2}(\tilde{\Omega})$-convergence). In conclusion we can pass to the limit in (3.18) (first in $m$ and then in $\varrho$ ) to obtain

$$
\tilde{E}_{t} \leq \tilde{E}_{s}+\frac{1}{2} \int_{s}^{t}\|\Phi\|_{L_{2}\left(\left(\mathfrak{U}, L^{2}\left(\mathbb{T}^{3}\right)\right)\right)}^{2} \mathrm{~d} t+\tilde{\mathscr{M}}_{t}-\tilde{\mathscr{M}}_{s}
$$

provided $t, s$ are Lebesgue points of $\tilde{E}_{t}=\frac{1}{2} \int_{\mathbb{T}^{3}}\left\langle\tilde{\nu}_{t, x},|\boldsymbol{\xi}|^{2}\right\rangle \mathrm{d} x+\frac{1}{2} \tilde{\lambda}_{t}\left(\mathbb{T}^{3}\right)$. Here we also used that $\frac{1}{\varrho} \tilde{\mathbb{E}} \tilde{\lambda}((t-$ $\left.\varrho, t) \times \mathbb{T}^{3}\right)$ stays bounded in $\varrho$ by $(3.18)$, which shows that $\tilde{\lambda}=\tilde{\lambda}_{t} \otimes \mathscr{L}^{1}$ with $\tilde{\lambda}_{t} \in L_{w^{*}}^{\infty}\left(0, T ; \mathscr{M}^{+}\left(\mathbb{T}^{3}\right)\right)$ $\tilde{\mathbb{P}}$-a.s. Relation (3.21) implies that the function

$$
t \mapsto \tilde{E}_{t}-\int_{0}^{t}\|\Phi\|_{L_{2}\left(\left(\mathfrak{U}, L^{2}\left(\mathbb{T}^{3}\right)\right)\right)}^{2} \mathrm{~d} \sigma-\tilde{\mathscr{M}}_{t}
$$

is non-increasing. Since it is also pathwise bounded (recall again (3.6)), left- and right-sided limits exist in all points. Furthermore, $\int_{0}^{*}\|\Phi\|_{L_{2}\left(\left(\mathfrak{U}, L^{2}\left(\mathbb{T}^{3}\right)\right)\right)}^{2} \mathrm{~d} \sigma$ and $\tilde{\mathscr{M}}$ are continuous such that left- and right-sided limits also exists for $\tilde{E}_{t}$. Approximating arbitrary $t$ and $s$ by Lebesgue points and using (3.21) we have

$$
\tilde{E}_{t^{+}} \leq \tilde{E}_{s^{-}}+\frac{1}{2} \int_{s}^{t}\|\Phi\|_{L_{2}\left(\left(\mathfrak{U}, L^{2}\left(\mathbb{T}^{3}\right)\right)\right)}^{2} \mathrm{~d} t+\tilde{\mathscr{M}}_{t}-\tilde{\mathscr{M}}_{s}
$$

$\tilde{\mathbb{P}}$-a.s. for all $t>s>0$. If $s=0$ we argue similarly to (3.18) but without the averaging in $s$. We obtain

$$
f_{t-\varrho}^{t} \tilde{E}_{r}^{\varepsilon_{m}} \mathrm{~d} r \leq \tilde{E}_{0}^{\varepsilon_{m}}+\frac{1}{2} f_{t-\varrho}^{t} \int_{0}^{r}\|\Phi\|_{L_{2}\left(\left(\mathfrak{U}, L^{2}\left(\mathbb{T}^{3}\right)\right)\right)}^{2} \mathrm{~d} \sigma \mathrm{d} r+f_{t-\varrho}^{t} \tilde{\mathscr{M}}_{r}^{\varepsilon_{m}} \mathrm{~d} r
$$

$\tilde{\mathbb{P}}$-a.s. provided $\varrho<t$. Since $E_{0}^{\varepsilon_{m}}=\frac{1}{2} \int_{\mathbb{T}^{3}}\left|\mathbf{u}_{0}^{\varepsilon_{m}}\right|^{2} \mathrm{~d} x$ we can argue again by Proposition 3.5 and (3.19) to conclude

$$
\tilde{E}_{t} \leq \tilde{E}_{0^{-}}+\frac{1}{2} \int_{0}^{t}\|\Phi\|_{L_{2}\left(\left(\mathfrak{U}, L^{2}\left(\mathbb{T}^{3}\right)\right)\right)}^{2} \mathrm{~d} \sigma+\tilde{\mathscr{M}}_{t}
$$

$\tilde{\mathbb{P}}$-a.s. for Lebesgue points $t$, where $\tilde{E}_{0^{-}}=\frac{1}{2} \int_{\mathbb{T}^{3}}\left|\tilde{\mathbf{u}}_{0}\right|^{2} \mathrm{~d} x$. Finally, we also obtain

$$
\tilde{E}_{t^{+}} \leq \tilde{E}_{0^{-}}+\frac{1}{2} \int_{0}^{t}\|\Phi\|_{L_{2}\left(\left(\mathfrak{U}, L^{2}\left(\mathbb{T}^{3}\right)\right)\right)}^{2} \mathrm{~d} \sigma+\tilde{\mathscr{M}}_{t}
$$

$\tilde{\mathbb{P}}$-a.s. for all $t>0$. This, in combination with (3.22), finishes the proof of the energy inequality (3.5). The proof of Theorem 3.3 is hereby complete. 


\section{Weak-Strong Uniqueness}

In this section we compare the dissipative solution from Definition 3.1 with a strong solution. The results are reminiscent of those from [3] on the compressible Navier-Stokes system. A strong solution to the stochastic Euler equations is known to exists at least in short time. A concept which we make precise in the following.

Definition 4.1. Let $\left(\Omega, \mathfrak{F},\left(\mathfrak{F}_{t}\right), \mathbb{P}\right)$ be a stochastic basis with a complete right-continuous filtration, let $W$ be an $\left(\mathfrak{F}_{t}\right)$-cylindrical Wiener process. A random variable $\mathbf{u}$ and a stopping time $\mathfrak{t}$ is called a (local) strong solution to system (3.1) provided

(a) the process $t \mapsto \mathbf{u}(t \wedge \mathfrak{t}, \cdot)$ is $\left(\mathfrak{F}_{t}\right)$-adapted, $\mathbf{u}(t \wedge \mathfrak{t}, \cdot), \nabla \mathbf{u}(t \wedge \mathfrak{t}, \cdot) \in C_{\text {loc }}\left([0, \infty) \times \mathbb{T}^{3}\right) \mathbb{P}$-a.s. and for all $T>0$

$$
\mathbb{E}\left[\sup _{0 \leq t \leq T}\left(\|\nabla \mathbf{u}(\cdot \wedge \mathfrak{t})\|_{L_{x}^{\infty}}+\|\mathbf{u}(\cdot \wedge \mathfrak{t})\|_{L_{x}^{\infty}}\right)\right]<\infty
$$

(b) for all $\varphi \in C_{\mathrm{div}}^{\infty}\left(\mathbb{T}^{3}\right)$ and all $t \geq 0$ there holds $\mathbb{P}$-a.s.

$$
\begin{aligned}
\int_{\mathbb{T}^{3}} \mathbf{u}(t \wedge \mathfrak{t}) \cdot \varphi \mathrm{d} x= & \int_{\mathbb{T}^{3}} \mathbf{u}(0) \cdot \varphi \mathrm{d} x-\int_{0}^{t \wedge \mathfrak{t}} \int_{\mathbb{T}^{3}}(\nabla \mathbf{u}) \mathbf{u} \cdot \varphi \mathrm{d} x \mathrm{~d} s \\
& +\int_{0}^{t \wedge \mathfrak{t}} \int_{\mathbb{T}^{3}} \varphi \cdot \Phi \mathrm{d} x \mathrm{~d} W
\end{aligned}
$$

(c) we have $\operatorname{div} \mathbf{u}(\cdot \wedge \mathfrak{t})=0 \mathbb{P}$-a.s.

Remark 4.2. A direct application of Itô's formula (in the Hilbert space version for $L_{\mathrm{div}}^{2}\left(\mathbb{T}^{3}\right)$ ) shows that strong solutions satisfy the energy equality

$$
\int_{\mathbb{T}^{3}}|\mathbf{u}(t)|^{2} \mathrm{~d} x=\int_{\mathbb{T}^{3}}|\mathbf{u}(0)|^{2} \mathrm{~d} x+2 \int_{0}^{t} \int_{\mathbb{T}^{3}} \mathbf{u} \cdot \Phi \mathrm{d} x \mathrm{~d} W+\int_{0}^{t}\|\Phi\|_{L_{2}\left(\mathfrak{U}, L^{2}\left(\mathbb{T}^{3}\right)\right)}^{2} \mathrm{~d} \sigma
$$

for all $t \in[0, \mathfrak{t}] \mathbb{P}$-a.s.

The existence of local-in-time strong solutions to (3.1) (however, under slip boundary conditions and not in the periodic setting) in the sense of Definition 4.1 was established in [23, Theorem 4.3] under certain assumptions imposed on the coefficient $\Phi$.

\subsection{Pathwise Weak-Strong Uniqueness}

We begin with the case that the dissipative solution and the strong solution are defined on the same probability space. We have the following result concerning weak-strong uniqueness.

Theorem 4.3. The pathwise weak-strong uniqueness holds true for the stochastic Euler equations (3.1) in the following sense: let

$$
\left(\left(\Omega, \mathfrak{F},\left(\mathfrak{F}_{t}\right), \mathbb{P}\right), \mathbf{u}, \mathcal{V}, W\right)
$$

be a dissipative martingale solution to (3.1) in the sense of Definition 3.1 and let $\mathbf{v}$ and a stopping time $\mathfrak{t}$ be a strong solution of the same problem in the sense of Definition 4.1 defined on the same stochastic basis with the same Wiener process and with the same initial data (meaning $\mathbf{v}(0, \cdot)=\mathbf{u}(0, \cdot) \mathbb{P}$-a.s.). Then we have for a.a. $(t, x)$ that $\mathbf{u}(t \wedge \mathfrak{t}, x)=\mathbf{v}(t \wedge \mathfrak{t}, x)$ and $\left(\nu_{t \wedge \mathfrak{t}, x}, \nu_{t \wedge \mathfrak{t}, x}^{\infty}, \lambda\right)=\left(\delta_{\mathbf{u}(t \wedge \mathfrak{t}, x)}, 0,0\right) \mathbb{P}$-a.s.

Proof. We start by introducing the stopping time

$$
\tau_{L}=\inf \left\{t \in(0, \mathfrak{t}) \mid\|\nabla \mathbf{v}(t, \cdot)\|_{L_{x}^{\infty}}>L\right\}, \quad L>0,
$$


and define $\tau_{L}=\mathfrak{t}$ if $\{\ldots\}=\emptyset$. Since $\mathbb{E}\left[\sup _{t \in[0, t]}\|\nabla \mathbf{v}(t)\|_{L_{x}^{\infty}}\right]<\infty$ by assumption (recall Definition 4.1) we have

$$
\mathbb{P}\left[\tau_{L}<\mathfrak{t}\right] \leq \mathbb{P}\left[\sup _{t \in[0, t]}\|\nabla \mathbf{v}(t)\|_{L_{x}^{\infty}} \geq L\right] \leq \frac{1}{L} \mathbb{E}\left[\sup _{t \in[0, t]}\|\nabla \mathbf{v}(t)\|_{L_{x}^{\infty}}\right] \rightarrow 0
$$

as $L \rightarrow \infty$ by Tschebyscheff's inequality. Consequently, we have

$$
\tau_{L} \rightarrow \mathfrak{t} \text { in probability. }
$$

Whence it is enough to show the claim in $\left(0, \tau_{L}\right)$ for a fixed $L$. We consider the functional

$$
F(t)=\frac{1}{2} \int_{\mathbb{T}^{3}}\left\langle\nu_{t, x},|\boldsymbol{\xi}-\mathbf{v}|^{2}\right\rangle \mathrm{d} x+\frac{1}{2} \lambda_{t}\left(\mathbb{T}^{3}\right)
$$

defined for a.a. $t<\mathfrak{t}$. Noting that $\mathbf{u}=\left\langle\nu_{t, x}, \boldsymbol{\xi}\right\rangle$ we can write

$$
\begin{aligned}
F(t) & =\frac{1}{2}\left(\int_{\mathbb{T}^{3}}\left\langle\nu_{t, x},|\boldsymbol{\xi}|^{2}\right\rangle+\lambda_{t}\left(\mathbb{T}^{3}\right)-2 \int_{\mathbb{T}^{3}} \mathbf{u} \cdot \mathbf{v} \mathrm{d} x+\int_{\mathbb{T}^{3}}|\mathbf{v}|^{2} \mathrm{~d} x\right), \\
& =E(t)+\frac{1}{2} \int_{\mathbb{T}^{3}}|\mathbf{v}|^{2} \mathrm{~d} x-\int_{\mathbb{T}^{3}} \mathbf{u} \cdot \mathbf{v} \mathrm{d} x .
\end{aligned}
$$

This definition can be extended to any $t<\mathfrak{t}$ by setting

$$
F(t)=E\left(t^{+}\right)+\frac{1}{2} \int_{\mathbb{T}^{3}}|\mathbf{v}|^{2} \mathrm{~d} x-\int_{\mathbb{T}^{3}} \mathbf{u} \cdot \mathbf{v} \mathrm{d} x
$$

recalling that $\mathbf{u}$ and $\mathbf{v}(\cdot \wedge \mathfrak{t})$ belong to $C_{w}\left([0, T] ; L^{2}\left(\mathbb{T}^{3}\right)\right)$. Taking the expectation of $F\left(t \wedge \tau_{L}\right)$ and using (3.5) and (4.1) yields

$$
\begin{aligned}
\mathbb{E}\left[F\left(t \wedge \tau_{L}\right)\right] & =\mathbb{E}\left[E\left(\left(t \wedge \tau_{L}\right)^{+}\right)\right]+\frac{1}{2} \mathbb{E} \int_{\mathbb{T}^{3}}\left|\mathbf{v}\left(t \wedge \tau_{L}\right)\right|^{2} \mathrm{~d} x-\mathbb{E} \int_{\mathbb{T}^{3}} \mathbf{u}\left(t \wedge \tau_{L}\right) \cdot \mathbf{v}\left(t \wedge \tau_{L}\right) \mathrm{d} x \\
& \leq \mathbb{E}\left(\int_{\mathbb{T}^{3}}|\mathbf{v}(0)|^{2} \mathrm{~d} x+\int_{0}^{t \wedge \tau_{L}}\|\Phi\|_{L_{2}\left(\mathfrak{U}, L^{2}\left(\mathbb{T}^{3}\right)\right)}^{2} \mathrm{~d} \sigma\right)-\mathbb{E} \int_{\mathbb{T}^{3}} \mathbf{u}\left(t \wedge \tau_{L}\right) \cdot \mathbf{v}\left(t \wedge \tau_{L}\right) \mathrm{d} x,
\end{aligned}
$$

where we also used $\mathbf{u}(0)=\mathbf{v}(0)$. Re-writing the last term using Lemma 2.4, we infer that

$$
\begin{aligned}
\mathfrak{A}:= & \int_{\mathbb{T}^{3}} \mathbf{u}\left(t \wedge \tau_{L}\right) \cdot \mathbf{v}\left(t \wedge \tau_{L}\right) \mathrm{d} x \\
= & \int_{\mathbb{T}^{3}} \mathbf{u}(0) \cdot \mathbf{v}(0) \mathrm{d} x+\int_{0}^{t \wedge \tau_{L}} \int_{\mathbb{T}^{3}}\left\langle\nu_{t, x}, \boldsymbol{\xi} \otimes \boldsymbol{\xi}\right\rangle: \nabla \mathbf{v} \mathrm{d} x \mathrm{~d} \sigma \\
& +\int_{\left(0, t \wedge \tau_{L}\right) \times \mathbb{T}^{3}}\left\langle\nu_{t, x}^{\infty}, \boldsymbol{\xi} \otimes \boldsymbol{\xi}\right\rangle: \nabla \mathbf{v} \mathrm{d} \lambda+\int_{0}^{t \wedge \tau_{L}} \int_{\mathbb{T}^{3}}(\mathbf{v}+\mathbf{u}) \cdot \Phi \mathrm{d} x \mathrm{~d} W \\
& +\int_{0}^{t \wedge \tau_{L}} \int_{\mathbb{T}^{3}} \operatorname{div}(\mathbf{v} \otimes \mathbf{v}) \cdot \mathbf{u} \mathrm{d} x \mathrm{~d} \sigma+\int_{0}^{t \wedge \tau_{L}}\|\Phi\|_{L_{2}\left(\mathfrak{U}, L^{2}\left(\mathbb{T}^{3}\right)\right)}^{2} \mathrm{~d} t .
\end{aligned}
$$

The stochastic term in $\mathfrak{A}$ vanishes upon computing expectations. Using also $\mathbf{u}(0)=\mathbf{v}(0)$ we obtain

$$
\mathbb{E}\left[F\left(t \wedge \tau_{L}\right)\right] \leq-\mathbb{E}\left(\mathfrak{A}_{I}+\mathfrak{A}_{I I}+\mathfrak{A}_{I I I}\right)
$$

with the remaining terms

$$
\begin{aligned}
\mathfrak{A}_{I} & =\int_{0}^{t \wedge \tau_{L}} \int_{\mathbb{T}^{3}}\left\langle\nu_{t, x}, \boldsymbol{\xi} \otimes \boldsymbol{\xi}\right\rangle: \nabla \mathbf{v} \mathrm{d} x \mathrm{~d} \sigma, \\
\mathfrak{A}_{I I} & =\int_{\left(0, t \wedge \tau_{L}\right) \times \mathbb{T}^{3}}\left\langle\nu_{t, x}^{\infty}, \boldsymbol{\xi} \otimes \boldsymbol{\xi}\right\rangle: \nabla \mathbf{v} \mathrm{d} \lambda, \\
\mathfrak{A}_{I I I} & =\int_{0}^{t \wedge \tau_{L}} \int_{\mathbb{T}^{3}} \operatorname{div}(\mathbf{v} \otimes \mathbf{v}) \cdot \mathbf{u} \mathrm{d} x \mathrm{~d} \sigma .
\end{aligned}
$$


Using standard identities for the nonlinear term we can write

$$
\mathfrak{A}_{I}+\mathfrak{A}_{I I I}=\int_{0}^{t \wedge \tau_{L}} \int_{\mathbb{T}^{3}}\left\langle\nu_{t, x},(\boldsymbol{\xi}-\mathbf{v}) \otimes(\boldsymbol{\xi}-\mathbf{v})\right\rangle: \nabla \mathbf{v} \mathrm{d} x \mathrm{~d} \sigma
$$

such that

$$
\begin{aligned}
\mathbb{E}\left[F\left(t \wedge \tau_{L}\right)\right] \leq & -\mathbb{E} \int_{0}^{t \wedge \tau_{L}} \int_{\mathbb{T}^{3}}\left\langle\nu_{t, x},(\boldsymbol{\xi}-\mathbf{v}) \otimes(\boldsymbol{\xi}-\mathbf{v})\right\rangle: \nabla \mathbf{v} \mathrm{d} x \mathrm{~d} \sigma \\
& -\mathbb{E} \int_{\left(0, t \wedge \tau_{L}\right) \times \mathbb{T}^{3}}\left\langle\nu_{t, x}^{\infty}, \boldsymbol{\xi} \otimes \boldsymbol{\xi}\right\rangle: \nabla \mathbf{v} \mathrm{d} \lambda \\
\leq & \mathbb{E} \int_{0}^{t \wedge \tau_{L}} \int_{\mathbb{T}^{3}}\left\langle\nu_{t, x},|\boldsymbol{\xi}-\mathbf{v}|^{2}\right\rangle|\nabla \mathbf{v}| \mathrm{d} x \mathrm{~d} \sigma+\mathbb{E} \int_{0}^{t \wedge \tau_{L}} \int_{\mathbb{T}^{3}}|\nabla \mathbf{v}| \mathrm{d} \lambda_{\sigma} \mathrm{d} \sigma \\
\leq & 2 \mathbb{E} \int_{0}^{t \wedge \tau_{L}} F(\sigma)\|\nabla \mathbf{v}\|_{L_{x}^{\infty}} \mathrm{d} \sigma \leq 2 L \mathbb{E} \int_{0}^{t \wedge \tau_{L}} F(\sigma) \mathrm{d} \sigma
\end{aligned}
$$

by definition of $\tau_{L}$. Finally, Gronwall's lemma implies that $\mathbb{E}\left[F\left(t \wedge \tau_{L}\right)\right]=0$ for a.e. $\mathrm{t}$ as required. Using (4.2) we obtain $F(t \wedge \mathfrak{t})=0 \mathbb{P}$-a.s. This finally yields the claim by definition of $F$.

Remark 4.4. Suppose that $\mathfrak{t}=T$ is deterministic. As can be seen from the proof, in this case the conclusion of Theorem 4.3 can be slightly strengthened to $\mathbf{u}=\mathbf{v}$ and $\mathcal{V}=\left(\delta_{\mathbf{u}}, 0,0\right) \mathbb{P}$-a.s., that is

$$
\begin{aligned}
& \mathbb{P}\left(\left\{\mathbf{u}(t, x)=\mathbf{v}(t, x) \text { for a.a. }(t, x) \in Q_{T}\right\}\right)=1, \\
& \mathbb{P}\left(\left\{\left(\nu_{t, x}, \nu_{t, x}^{\infty}, \lambda\right)=\left(\delta_{\mathbf{u}(t, x)}, 0,0\right) \text { for a.a. }(t, x) \in Q_{T}\right\}\right)=1 .
\end{aligned}
$$

\subsection{Weak-Strong Uniqueness in Law}

In this subsection we are finally concerned with the case that the dissipative solution and the strong solution are defined on distinct probability spaces. We obtain the following result.

Theorem 4.5. The weak-strong uniqueness in law holds true for the stochastic Euler equations (3.1) in the following sense: Let

$$
\left[\left(\Omega^{1}, \mathfrak{F}^{1},\left(\mathfrak{F}_{t}^{1}\right)_{t \geq 0}, \mathbb{P}^{1}\right), \mathbf{u}^{1}, \mathcal{V}^{1}, W^{1}\right]
$$

be a dissipative martingale solution to (3.1) in the sense of Definition 3.1 and let $\mathbf{u}^{2}$ be a strong solution of the same problem in the sense of Definition 4.1 (with $\mathfrak{t}=T$ deterministic) defined on a stochastic basis $\left(\Omega^{2}, \mathfrak{F}^{2},\left(\mathfrak{F}_{t}^{2}\right)_{t \geq 0}, \mathbb{P}^{2}\right)$ with the Wiener process $W^{2}$. Suppose that

$$
\mathbb{P}^{1} \circ\left(\mathbf{u}^{1}(0)\right)^{-1}=\mathbb{P}^{2} \circ\left(\mathbf{u}^{2}(0)\right)^{-1},
$$

then

$$
\mathbb{P}^{1} \circ\left(\mathbf{u}^{1}, \mathcal{V}^{1}\right)^{-1}=\mathbb{P}^{2} \circ\left(\mathbf{u}^{2},\left(\delta_{\mathbf{u}^{2}(t, x), 0,0}\right)\right)^{-1}
$$

Proof. Let us assume that

$$
\left[\left(\Omega^{1}, \mathfrak{F}^{1},\left(\mathfrak{F}_{t}^{1}\right)_{t \geq 0}, \mathbb{P}^{1}\right), \mathbf{u}^{1}, \mathcal{V}^{1}, W^{1}\right]
$$

is a dissipative martingale solution to (3.1) in the sense of Definition 3.1 and let $\mathbf{u}^{2}$ be a strong solution of the same problem in the sense of Definition 4.1 (with $\mathfrak{t}=T$ ). Different to Theorem $4.3 \mathbf{u}^{2}$ is now defined on a distinct stochastic basis $\left(\Omega^{2}, \mathfrak{F}^{2},\left(\mathfrak{F}_{t}^{2}\right)_{t \geq 0}, \mathbb{P}^{2}\right)$ with a distinct Wiener process $W^{2}$. We set $\mathbf{v}^{j}=\mathbf{u}^{j}-\mathbf{u}^{j}(0)$ for $t \geq 0$ and $j=1,2$. We consider the topological space

$$
\mathcal{X}_{T}:=C\left([0, T] ; W_{\operatorname{div}}^{-4,2}\left(\mathbb{T}^{3}\right)\right) \cap C_{w}\left([0, T] ; L_{\operatorname{div}}^{2}\left(\mathbb{T}^{3}\right)\right) \times Y_{2}\left(Q_{T}\right) \times C\left([0, T], \mathfrak{U}_{0}\right)
$$

together with the $\sigma$-algebra $\mathscr{B} \mathcal{X}_{T}$ defined in analogy to (3.3). Setting

$$
\Theta=L^{2}\left(\mathbb{T}^{3}\right) \times \mathcal{X}_{T}, \quad \mathscr{B}_{\Theta}=\mathscr{B}\left(L^{2}\left(\mathbb{T}^{3}\right)\right) \otimes \mathscr{B}_{\mathcal{X}_{T}},
$$


we denote the probability law $\mathcal{L}\left[\mathbf{u}^{j}(0), \mathbf{v}^{j}, \mathcal{V}^{j}, W^{j}\right]$ on $\left(\Theta, \mathscr{B}_{\Theta}\right)$ by $\mu^{j}$ (recall that $\mathcal{V}^{2}=\left(\delta_{\mathbf{u}^{2}(t, x), 0,0}\right)$ for the strong solution). It satisfies

$$
\mu^{j}(\mathscr{A})=\mathbb{P}^{j}\left(\left[\mathbf{u}^{j}(0), \mathbf{v}^{j}, \mathcal{V}^{j}, W^{j}\right] \in \mathscr{A}\right), \quad \mathscr{A} \in \mathscr{B}_{\Theta} .
$$

The generic element of $\Theta$ is denoted by $\theta=\left(\tilde{\mathbf{u}}_{0}, \tilde{W}, \tilde{\mathbf{v}}, \tilde{\mathcal{V}}\right)$. The marginal of each $\mathbb{P}^{j}$ on the $\tilde{\mathbf{u}}_{0}$-coordinate is $\Lambda$, the marginal on the $\tilde{W}$-coordinate is the Wiener measure $\mathbb{P}_{*}$ and the distribution of the pair is the product measure $\Lambda \otimes \mathbb{P}_{*}$ because $\mathbf{u}_{0}^{j}$ is $\mathfrak{F}_{0}^{j}$-measurable and $W^{j}$ is independent of $\mathfrak{F}_{0}^{j}$. Moreover, under $\mathbb{P}^{j}$ the initial value of the $\tilde{\mathbf{v}}$-coordinate is zero a.s.

In a first step we are going to construct a product probability space. In order to do this we need regular conditional probabilities and in the following we argue why this is possible in our situation. Let $(\mathcal{O}, \mathscr{Y})$ be a measure space, where $\mathcal{O}$ is a Hausdorff topological space and $\mathscr{Y}$ is countably generated. Let $\mathcal{U}$ be a regular probability measure on $(\mathcal{O}, \mathscr{Y})$, i.e.

$$
\mathcal{U}(A)=\sup \{\mathcal{U}(K): K \in \mathscr{Y}, K \subset A \text { compact }\} \quad \forall A \in \mathscr{Y} .
$$

It is well-known that under these assumptions there is a regular conditional probability for $\mathcal{U}$, see e.g. [24, introduction]. Since $\mathcal{X}$ is a quasi-Polish space and $L^{2}\left(\mathbb{T}^{3}\right)$ is a Banach space it is clear that $\Theta$ is Hausdorff. We have to argue that $B_{\Theta}$ is countable generated. It is clear that $\left(\mathscr{B}\left(C\left([0, T] ; W_{\text {div }}^{-4,2}\left(\mathbb{T}^{3}\right)\right)\right.\right.$ and $\mathscr{B}\left(C\left([0, T], \mathfrak{U}_{0}\right)\right)$ are countably generated since the spaces in question are both Polish. As far as $\mathscr{B}_{T}\left(C_{w}\left([0, T] ; L_{\text {div }}^{2}\left(\mathbb{T}^{3}\right)\right)\right)$ is concerned we refer to [10, Section 4] for a corresponding statement. Finally, since the function $f_{n}$ from (2.1) range in the Polish space $[-1,1]$ and are continuous we have that $\sigma\left(f_{n}\right)$ is countably generated for each $n \in \mathbb{N}$. Since the family $\left\{f_{n}\right\}$ is countable we conclude that $\mathscr{B}_{Y}$ defined in (2.5) is countably generated. In conclusion there is a regular conditional probability ${ }^{3}$

$$
Q_{j}\left(\tilde{\mathbf{u}}_{0}, \tilde{W}, \mathscr{A}\right): L^{2}\left(\mathbb{T}^{3}\right) \times C\left([0, T], \mathfrak{U}_{0}\right) \times \mathscr{B}_{\mathbf{u}} \otimes \mathscr{B}_{Y} \rightarrow[0,1]
$$

such that

(i) For each $\left(\tilde{\mathbf{u}}_{0}, \tilde{W}\right) \in L^{2}\left(\mathbb{T}^{3}\right) \times C\left([0, T], \mathfrak{U}_{0}\right)$ we have that

$$
Q_{j}(\mathbf{w}, B, \cdot):\left(C\left([0, T] ; W_{\operatorname{div}}^{-4,2}\left(\mathbb{T}^{3}\right) \cap C_{w}\left([0, T] ; L^{2}\left(\mathbb{T}^{3}\right)\right) \times Y_{2}\left(Q_{T}\right) ; \mathscr{B}_{\mathbf{u}} \otimes \mathscr{B}_{Y}\right) \rightarrow[0,1]\right.
$$

is a probability measure;

(ii) The mapping $\left(\tilde{\mathbf{u}}_{0}, \tilde{W}\right) \rightarrow Q_{j}\left(\tilde{\mathbf{u}}_{0}, \tilde{W}, \mathscr{A}\right)$ is $\mathscr{B}\left(L^{2}\left(\mathbb{T}^{3}\right)\right) \otimes \mathscr{B}\left(C\left([0, T], \mathfrak{U}_{0}\right)\right)$ measurable for each $\mathscr{A} \in$ $\mathscr{B}_{\mathbf{u}} \otimes \mathscr{B}_{Y}$

(iii) We have that

$$
\mu^{j}(G \times \mathscr{A})=\int_{G} Q_{j}\left(\tilde{\mathbf{u}}_{0}, \tilde{W}, \mathscr{A}\right) \mathrm{d} \Lambda\left(\tilde{\mathbf{u}}_{0}\right) \mathrm{d} \mathbb{P}_{*}(\tilde{W}), \quad \mathscr{A} \in \mathscr{B}_{\mathbf{u}} \otimes \mathscr{B}_{Y},
$$

for all $G \in \mathscr{B}\left(L^{2}\left(\mathbb{T}^{3}\right)\right) \otimes \mathscr{B}\left(C\left([0, T], \mathfrak{U}_{0}\right)\right.$.

Finally, we define

$$
\tilde{\Omega}=\Theta \times C\left([0, T] ; W_{\operatorname{div}}^{-4,2}\left(\mathbb{T}^{3}\right)\right) \cap C_{w}\left([0, T] ; L_{\operatorname{div}}^{2}\left(\mathbb{T}^{3}\right)\right) \times Y_{2}\left(Q_{T}\right)
$$

and $\tilde{\mathfrak{F}}$ is the completion of $\mathscr{B}_{\Theta} \otimes \mathscr{B}_{\mathbf{u}} \otimes \mathscr{B}_{Y}$ with respect to the probability measure

$$
\tilde{\mathbb{P}}\left(G \times \mathscr{A}_{1} \times \mathscr{A}_{2}\right)=\int_{G} Q_{1}\left(\tilde{\mathbf{u}}_{0}, \tilde{W}, \mathscr{A}_{1}\right) Q_{2}\left(\tilde{\mathbf{u}}_{0}, \tilde{W}, \mathscr{A}_{2}\right) \mathrm{d} \Lambda\left(\tilde{\mathbf{u}}_{0}\right) \mathrm{d} \mathbb{P}_{*}(\tilde{W})
$$

for $\mathscr{A}_{1}, \mathscr{A}_{2} \in \mathscr{B}_{\mathbf{u}} \otimes \mathscr{B}_{Y}$ and $G \in \mathscr{B}\left(L^{2}\left(\mathbb{T}^{3}\right)\right) \otimes \mathscr{B}\left(C\left([0, T], \mathfrak{U}_{0}\right)\right.$. The space $(\tilde{\Omega}, \tilde{\mathfrak{F}}, \tilde{\mathbb{P}})$ is the product probability space we were seeking and we obtain for $j=1,2$

$$
\tilde{\mathbb{P}}\left(\left\{\tilde{\omega}=\left(\tilde{\mathbf{u}}_{0}, \tilde{W}, \tilde{\mathbf{v}}^{1}, \tilde{\mathcal{V}}^{1}, \tilde{\mathbf{v}}^{2}, \tilde{\mathcal{V}}^{2}\right) \in \tilde{\Omega}:\left(\tilde{\mathbf{u}}_{0}, \tilde{W}, \tilde{\mathbf{v}}^{j}, \tilde{\mathcal{V}}^{j}\right) \in \mathscr{A}\right\}\right)=\mu_{j}(\mathscr{A}), \quad \mathscr{A} \in \mathscr{B}_{\Theta} .
$$

\footnotetext{
${ }^{3}$ In fact, from [24] we deduce a conditional probability defined on $L^{2}\left(\mathbb{T}^{3}\right) \times C\left([0, T], \mathfrak{U}_{0}\right) \times \mathscr{B}_{\Theta}$. Restricting the $\sigma$-field to events of the form $L_{\text {div }}^{2}\left(\mathbb{T}^{3}\right) \otimes \mathscr{A} \otimes C\left([0 ; T] ; \mathfrak{U}_{0}\right)$ yields the object in question.
} 
Finally, we define the filtrations

$$
\begin{aligned}
& \tilde{\mathfrak{F}}_{t}^{j}=\sigma\left(\sigma\left(\tilde{\mathbf{u}}_{0}, \mathbf{r}_{t} \tilde{W}, \mathbf{r}_{t} \tilde{\mathbf{v}}^{j}, \mathbf{r}_{t} \tilde{\mathcal{V}}^{j}\right) \cup \sigma_{t}\left[\tilde{\mathcal{V}}^{j}\right] \cup\{\mathcal{N} \in \tilde{\mathfrak{F}} ; \tilde{\mathbb{P}}(\mathcal{N})=0\}\right), j=1,2, \\
& \tilde{\mathfrak{F}}_{t}=\sigma\left(\sigma\left(\tilde{\mathbf{u}}_{0}, \mathbf{r}_{t} \tilde{W}, \mathbf{r}_{t} \tilde{\mathbf{v}}^{1}, \mathbf{r}_{t} \tilde{\mathbf{v}}^{2}\right) \cup \sigma_{t}\left[\tilde{\mathcal{V}}^{1}\right] \cup \sigma_{t}\left[\tilde{\mathcal{V}}^{2}\right] \cup\{\mathcal{N} \in \tilde{\mathfrak{F}} ; \tilde{\mathbb{P}}(\mathcal{N})=0\}\right),
\end{aligned}
$$

which ensure the correct measurabilities. Here $\sigma_{t}$ denotes the history of a random distribution as defined in (2.6), where generalised Young measures are identified as random distribution in the sense of (2.4).

In the next step we aim to show that for $j=1,2$

$$
\left[\left(\Omega, \tilde{\mathfrak{F}},\left(\tilde{\mathfrak{F}}_{t}\right)_{t \geq 0}, \tilde{\mathbb{P}}\right), \tilde{\mathbf{v}}^{j}+\tilde{\mathbf{u}}_{0}, \tilde{\mathcal{V}}^{j}, \tilde{W}\right]
$$

is a dissipative martingale solution to (3.1) in the sense of Definition 3.1 and that $\mathbf{v}^{2}+\mathbf{u}_{0}$ is a strong solution. As in (3.11) we can prove that $\tilde{\mathbb{P}}$-a.s.

$$
\tilde{\mathbf{v}}^{j}(t, x)+\tilde{\mathbf{u}}_{0}(x)=\left\langle\tilde{\nu}_{t, x}^{j}, \boldsymbol{\xi}\right\rangle \text { for a.a. }(t, x) \in Q_{T},
$$

where $\mathcal{V}^{j}=\left(\tilde{\nu}_{t, x}^{j}, \tilde{\nu}_{t, x}^{\infty, j}, \tilde{\lambda}^{j}\right)$. Defining the functional

$$
\begin{aligned}
\mathfrak{M}(\mathbf{w}, \mathcal{V})_{t}= & \int_{\mathbb{T}^{3}} \mathbf{w}(t) \cdot \boldsymbol{\varphi} \mathrm{d} x-\int_{0}^{t} \int_{\mathbb{T}^{3}}\left\langle\nu_{t, x}, \boldsymbol{\xi} \otimes \boldsymbol{\xi}\right\rangle: \nabla \boldsymbol{\varphi} \mathrm{d} x \mathrm{~d} s \\
& -\int_{(0, t) \times \mathbb{T}^{3}}\left\langle\nu_{t, x}^{\infty}, \boldsymbol{\xi} \otimes \boldsymbol{\xi}\right\rangle: \nabla \boldsymbol{\varphi} \mathrm{d} \lambda, \quad \boldsymbol{\varphi} \in C_{\mathrm{div}}^{\infty}\left(\mathbb{T}^{3}\right),
\end{aligned}
$$

we can argue as in Sect. 3.3 to prove that $\mathfrak{M}\left(\tilde{\mathbf{u}}^{j}+\tilde{\mathbf{u}}_{0}, \mathcal{V}^{j}\right)$ is an $\left(\tilde{\mathfrak{F}}_{t}^{j}\right)$-martingale. Moreover its quadratic variation and cross variation with respect to $\tilde{W}$ are given by $\mathfrak{N}$ and $\mathfrak{N}^{k}$ respectively. Consequently, both solutions satisfy the momentum equation in the sense of Definition 3.1 (g) driven by $\tilde{W}$. Finally, we can use again Proposition 2.5 to argue that the energy inequality continues to hold on the product probability space following the arguments of Sect.3.3.

In order to apply our pathwise weak-strong uniqueness result from Theorem 4.3 it suffices to argue that $\tilde{\mathbf{u}}^{2}=\tilde{\mathbf{v}}^{2}+\tilde{\mathbf{u}}_{0}$ is a strong solution. On the original probability space $\left(\Omega^{2}, \mathfrak{F}^{2}, \mathbb{P}^{2}\right)$ the strong solution $\mathbf{u}^{2}$ is supported on $C\left([0, T] ; C^{1}\left(\mathbb{T}^{3}\right)\right)$ and we have $\mathcal{V}^{2}=\left(\delta_{\mathbf{u}^{2}(t, x)}, 0,0\right) \mathbb{P}^{2}$-a.s. The embedding

$$
C\left([0, T] ; C^{1}\left(\mathbb{T}^{3}\right)\right) \hookrightarrow C\left([0, T] ; W^{-4,2}\left(\mathbb{T}^{3}\right)\right)
$$

is continuous and dense such that

$$
C\left([0, T] ; C^{1}\left(\mathbb{T}^{3}\right)\right) \in \mathscr{B}\left(C\left([0, T] ; W^{-4,2}\left(\mathbb{T}^{3}\right)\right)\right) \subset \mathscr{B}_{\mathbf{u}},
$$

cf. [34, Cor. A.2]. We conclude

$$
\mu_{2}\left(C\left([0, T] ; C^{1}\left(\mathbb{T}^{3}\right)\right)\right)=\mathbb{P}_{2}\left(\mathbf{v} \in C\left([0, T] ; C^{1}\left(\mathbb{T}^{3}\right)\right)\right)=1
$$

such that $\tilde{\mathbf{u}}^{2}$ is a strong solution on in the sense of Definition 4.1 (with $\mathfrak{t}=T$ ) on $(\tilde{\Omega}, \tilde{\mathfrak{F}}, \tilde{\mathbb{P}})$. Moreover, we have $\tilde{\mathbf{u}}^{2}(0)=\tilde{\mathbf{u}}_{0}=\tilde{\mathbf{u}}^{1}(0) \tilde{\mathbb{P}}_{\text {-a.s. }}$. We conclude by Theorem 4.3 (see also Remark 4.4) that $\tilde{\mathbb{P}}$-a.s.

$$
\left(\tilde{\mathbf{u}}^{1}, \tilde{\mathcal{V}}^{1}\right)=\left(\tilde{\mathbf{u}}^{2}, \tilde{\mathcal{V}}^{2}\right)=\left(\tilde{\mathbf{u}}^{2},\left(\delta_{\tilde{\mathbf{u}}^{2}}, 0,0\right)\right)
$$

Finally, we obtain

$$
\begin{aligned}
\mu_{1}(\mathscr{A}) & =\tilde{\mathbb{P}}\left(\left\{\tilde{\omega} \in \tilde{\Omega}:\left(\tilde{\mathbf{u}}_{0}, \tilde{W}, \tilde{\mathbf{v}}^{1}+\tilde{\mathbf{u}}_{0}, \tilde{\mathcal{V}}^{1}\right) \in \mathscr{A}\right\}\right) \\
& =\tilde{\mathbb{P}}\left(\left\{\tilde{\omega} \in \tilde{\Omega}:\left(\tilde{\mathbf{u}}_{0}, \tilde{W}, \tilde{\mathbf{v}}^{2}+\tilde{\mathbf{u}}_{0}, \tilde{\mathcal{V}}^{2}\right) \in \mathscr{A}\right\}\right)=\mu_{2}(\mathscr{A})
\end{aligned}
$$

for all $\mathscr{A} \in \mathscr{B}_{\Theta}$ which finishes the proof. 
Acknowledgements. The authors would like to thank M. Hofmanová, R. Zhu and X. Zhu for stimulating discussions and suggestions which helped to improve the paper. They are also grateful to the anomymous referee for the careful reading of the paper and the valuable comments. T.C. Moyo was supported by The Maxwell Institute Graduate School in Analysis and its Applications, a Centre for Doctoral Training funded by the UK Engineering and Physical Sciences Research Council (Grant EP/L016508/01), the Scottish Funding Council, Heriot-Watt University and the University of Edinburgh.

\section{Declarations}

Conflict of interest The authors declare that they have no conflict of interest.

Open Access. This article is licensed under a Creative Commons Attribution 4.0 International License, which permits use, sharing, adaptation, distribution and reproduction in any medium or format, as long as you give appropriate credit to the original author(s) and the source, provide a link to the Creative Commons licence, and indicate if changes were made. The images or other third party material in this article are included in the article's Creative Commons licence, unless indicated otherwise in a credit line to the material. If material is not included in the article's Creative Commons licence and your intended use is not permitted by statutory regulation or exceeds the permitted use, you will need to obtain permission directly from the copyright holder. To view a copy of this licence, visit http://creativecommons.org/licenses/by/4.0/.

Publisher's Note Springer Nature remains neutral with regard to jurisdictional claims in published maps and institutional affiliations.

\section{References}

[1] Alibert, J.J., Bouchitté, G.: Non-uniform integrability and generalized Young measures. J. Conv. Anal. 4, 129-147 (1997)

[2] Bessaih, H., Flandoli, F.: 2-D Euler equation perturbed by noise. Nonlinear Differ. Equ. Appl. 6, 35-54 (1999)

[3] Breit, D., Feireisl, E., Hofmanová, M.: Compressible fluids driven by stochastic forcing: the relative energy inequality and applications. Commun. Math. Phys. 350, 443-473 (2017)

[4] Breit, D., Feireisl, E., Hofmanová, M.: Stochastically Forced Compressible Fluid Flows. De Gruyter Series in Applied and Numerical Mathematics. De Gruyter, Berlin (2018)

[5] Breit, D., Feireisl, E., Hofmanová, M.: On solvability and ill-posedness of the compressible Euler system subject to stochastic forces. Anal. PDE 13, 371-402 (2020)

[6] Breit, D., Hofmanová, M.: Stochastic Navier-Stokes equations for compressible fluids. Indiana Univ. Math. J. 65, 1183$1250(2016)$

[7] Brenier, Y., De Lellis, C., Székelyhidi Jr., L.: Weak-strong uniqueness for measure-valued solutions. Commun. Math. Phys. 305, 351-361 (2011)

[8] Brzeźniak, Z., Motyl, E.: Existence of a martingale solution of the stochastic Navier-Stokes equations in unbounded 2D and 3D domains. J. Differ. Equ. 254, 1627-1685 (2013)

[9] Brzeźniak, Z., Ondreját, M.: Strong solutions to stochastic wave equations with values in Riemannian manifolds. J. Funct. Anal. 253, 449-481 (2007)

[10] Brzeźniak, Z., Ondreját, M., Seidler, J.: Invariant measures for stochastic nonlinear beam and wave equations. J. Differ. Equ. 260, 4157-4179 (2016)

[11] Brzeźniak, Z., Peszat, S.: Stochastic two dimensional Euler equations. Ann. Probab. 29(4), 1796-1832 (2001)

[12] Da Prato, G., Zabczyk, J.: Stochastic Equations in Infinite Dimensions. Encyclopedia of Mathematics and Its Applications, vol. 44. Cambridge University Press, Cambridge (1992)

[13] Capiński, M., Cutland, N.: Stochastic Navier-Stokes equations. Acta Appl. Math. 25(1), 59-85 (1991)

[14] Capinski, M., Cutland, N.J.: Stochastic Euler equations on the torus. Ann. Appl. Probab. 9, $688-705$ (1999)

[15] Debussche, A., Glatt-Holtz, N., Temam, R.: Local martingale and pathwise solutions for an abstract fluids model. Physica D 14-15, 1123-1144 (2011)

[16] De Lellis, C., Székelyhidi Jr., L.: The Euler equations as a differential inclusion. Ann. Math. 2(170), 1417-1436 (2009)

[17] De Lellis, C., Székelyhidi Jr., L.: On admissibility criteria for weak solutions of the Euler equations. Arch. Ration. Mech. Anal. 195, 225-260 (2010)

[18] DiPerna, R.J.: Measure-valued solutions of conservation laws. Arch. Ration. Mech. Anal. 88, 223-270 (1985)

[19] DiPerna, R.J., Majda, A.J.: Oscillations and concentrations in weak solutions of the incompressible fluid equations. Commun. Math. Phys. 108(4), 667-689 (1987)

[20] Eyink, G.: Exact results on stationary turbulence in 2D: consequences of vorticity conservation. Physica D 91(1), 97-142 (1996)

[21] Flandoli, F., Gątarek, D.: Martingale and stationary solutions for stochastic Navier-Stokes equations. Probab. Theory Relat. Fields 102, 367-391 (1995) 
[22] Flandoli, F., Romito, M.: Markov selections for the 3D stochastic Navier-Stokes equations. Probab. Theory Relat. Fields 140, 407-458 (2008)

[23] Glatt-Holtz, N.E., Vicol, V.C.: Local and global existence of smooth solutions for the stochastic Euler equations with multiplicative noise. Ann. Probab. 42(1), 80-145 (2014)

[24] Hoffmann-Jorgensen, J.: Existence of conditional probabilities. Math. Scad. 28, 257-264 (1971)

[25] Hoffmanová, M.: Degenerate parabolic stochastic partial differential equations. Stoch. Proc. Appl. 123(12), 4294-4336 (2013)

[26] Jakubowski, A.: The almost sure Skorokhod representation for subsequences in nonmetric spaces. Teor. Veroyatnost. i Primenen 42(1), 209-216 (1997); translation in Theory Probab. Appl. 42(1), 167-174 (1998)

[27] Kim, J.U.: On the stochastic Euler equations in a two-dimensional domain. SIAM J. Math. Anal. 33(5), 1211-1227 (2002)

[28] Kim, J.U.: Existence of a local smooth solution in probability to the stochastic Euler equations in $R^{3}$. J. Funct. Anal. 256, 3660-3687 (2009)

[29] Kim, J.U.: Measure valued solutions to the stochastic Euler equations in $\mathbb{R}^{d}$. Stoch. PDE: Anal. Comp. 3, 531-569 (2015)

[30] Kristensen, J., Rindler, F.: Characterization of generalized gradient young measures generated by sequences in $W^{1,1}$ and BV. Arch. Ration. Mech. Anal. 197, 539-598 (2010)

[31] Lions, P.L.: Mathematical Topics in Fluid Mechanics. Vol. 1. Incompressible Models. Oxford Lecture Series in Mathematics and Its Applications, 3. Oxford University Press, New York (1996)

[32] Mikulevicius, R., Valiukevicius, G.: On stochastic Euler equation in Rd. Electron. J. Probab. 5, 1-20 (2000)

[33] Novikov, E.: Functionals and the random-force method in turbulence theory. Sov. Phys. JETP 20, 1290-1294 (1965)

[34] Ondreját, M.: Stochastic nonlinear wave equations in local Sobolev spaces. Elect. J. Prob. 15, 1041-1091 (2010)

[35] Vishik, M., Komech, A., Fursikov, A.: Some mathematical problems of statistical hydromechanics. Uspekhi Mat. Nauk 34(5(209)):135-210, 256 (1979)

[36] E. Wiedemann. Existence of weak solutions for the incompressible Euler equations. Ann. Inst. H. Poincaré Anal. Non Linéaire 28, 727-730 (2011)

D. Breit and T. C. Moyo

Department of Mathematics

Heriot-Watt University

Riccarton Edinburgh EH14 4AS

UK

e-mail: db13@hw.ac.uk

(accepted: June 25, 2021; published online: July 15, 2021) 